\title{
ESTIMATES AND COMPUTATIONS FOR MELTING AND SOLIDIFICATION PROBLEMS *
}

\author{
James M. GReenberG ${ }^{1}$
}

\begin{abstract}
In this paper we focus on melting and solidification processes described by phase-field models and obtain rigorous estimates for such processes. These estimates are derived in Section 2 and guarantee the convergence of solutions to non-constant equilibrium patterns. The most basic results conclude with the inequality (2.31). The estimates in the remainder of Section 2 illustrate what obtains if the initial data is progressively more regular and may be omitted on first reading. We also present some interesting numerical simulations which demonstrate the equilibrium structures and the approach of the system to non-constant equilibrium patterns. The novel feature of these calculations is the linking of the small parameter in the system, $\delta$, to the grid spacing, thereby producing solutions with approximate sharp interfaces. Similar ideas have been used by Caginalp and Sokolovsky [5]. A movie of these simulations may be found at http: www.math.cmu.edu/math/people/greenberg.html
\end{abstract}

Mathematics Subject Classification. 35B25, 35B40, 35B45.

Received: September 14, 2000. Revised: January 25, 2001.

\section{INTRODUCTION}

In this paper we focus on melting and solidification processes described by phase-field models and obtain rigorous estimates for such processes. These estimates are derived in Section 2 and guarantee the convergence of solutions to non-constant equilibrium patterns. The most basic results conclude with the inequality (2.31). The estimates in the remainder of Section 2 illustrate what obtains if the initial data is progressively more regular and may be omitted on first reading. We also present some interesting numerical simulations which demonstrate the equilibrium structures and the approach of the system to non-constant equilibrium patterns. The novel feature of these calculations is the linking of the small parameter in the system, $\delta$, to the grid spacing, thereby producing solutions with approximate sharp interfaces. Similar ideas have been used by Caginalp and Sokolovsky [5].

For the definiteness we focus on the system

$$
T_{t}+\left(1-p^{2}\right) p_{t}-a^{2} \Delta T=0, \quad(x, y) \in \Omega
$$

\footnotetext{
Keywords and phrases. Phase-field models, melting and solidification.

* This research was partially supported by the Applied Mathematical Sciences Program, U.S. Department of Energy and the Mathematics and Computer Science Division, Army Research Office.

${ }^{1}$ Carnegie Mellon University, Department of Mathematical Sciences, Pittsburgh, PA 15213, USA.

e-mail: greenberg@andrew.cmu.edu
} 
and

$$
\alpha \delta p_{t}-\lambda^{2} \delta^{2} \Delta p=\mu T\left(1-p^{2}\right)+p\left(1-p^{2}\right) \quad, \quad(x, y) \in \Omega .
$$

Here $\Delta$ is the two-dimensional Laplacian, $T$ is the difference between the absolute temperature $\theta$ and the nominal melt temperature of the medium $\theta_{m}$; i.e. $T=\theta-\theta_{m}$, and $p$ is a nonconservative order parameter. The parameters $\alpha, a$, and $\lambda$ are positive and $O(1), 0<\mu \leq 1$, and $0<\delta \ll 1$. For definiteness we restrict our attention to the case where $\Omega$ is the unit square

$$
\Omega=\{(x, y) \mid-1 / 2<x, y<1 / 2\} .
$$

On $\partial \Omega$ we assume that

$$
\frac{\partial T}{\partial n}=\frac{\partial p}{\partial n}=0
$$

and at $t=0, T$ and $p$ are prescribed.

The reader should note that (1.1) is the balance of energy

$$
e_{t}+\operatorname{div} q=0
$$

where

$$
e=\theta_{m}+T+p-p^{3} / 3 \text { and } q=-a^{2} \nabla T .
$$

The reader may be concerned that as defined $e$ is not globally monotone increasing in the order parameter $p$ but this is really not an issue since (i) $e$ is monotone increasing in $p$ on $[-1,1]$ and (ii) if the initial data for $p$ satisfies $-1 \leq p\left(x, y, 0^{+}\right) \leq 1$, then the same estimate applies for all future times $t>0$. This last assertion is a direct consequence of (1.2) and the observation that at a local maximum (minimum) of $p$ that $\Delta p \leq 0(\Delta p \geq 0)$.

The system (1.1) and (1.2) is close to what Penrose and Fife [8,9] and Sekerka et al. [10] call a thermodynamically consistent system. If one lets $\theta$ denote the absolute temperature and $\theta_{m}$ denote the nominal melt temperature of the solid, then our internal energy, expressed as a function of $\theta$ and $p$, may be written as

$$
e=\theta+p-\frac{p^{3}}{3}
$$

and the Helmholtz free energy, $F(\theta, p)$, satisfies

$$
\theta^{2} \frac{\partial}{\partial \theta}\left(\frac{F}{\theta}\right)=-e=-\theta-\left(p-\frac{p^{3}}{3}\right)
$$

and

$$
F\left(\theta_{m}, p\right)=\frac{\left(1-p^{2}\right)^{2}}{4}
$$

These latter two conditions imply that

$$
F(\theta, p)=\frac{\theta}{4 \theta_{m}}\left(1-p^{2}\right)^{2}+\frac{1}{\theta_{m}} \ln \left(\frac{\theta_{m}}{\theta}\right)-\frac{\left(\theta-\theta_{m}\right)}{\theta_{m}}\left(p-p^{3} / 3\right)
$$

and the thermodynamically consistent phase-field equation for $p$ associated with $F$ is

$$
\tilde{\alpha} \delta p_{t}-\tilde{\lambda}^{2} \delta^{2} \Delta p=-\frac{1}{\theta} \frac{\partial F}{\partial p}=\left(\frac{\theta-\theta_{m}}{\theta \theta_{m}}+\frac{p}{\theta_{m}}\right)\left(1-p^{2}\right) .
$$


where $\tilde{\alpha}=\frac{\alpha}{\theta_{m}}$ and $\tilde{\lambda}=\frac{\lambda}{\left(\theta_{m}\right)^{1 / 2}}$. This latter equation, together with $\theta=\theta_{m}+T$, implies that the above consistent phase-field equation reduces to (1.2) with $\mu=\frac{1}{\theta_{m}}$ in the limit where $T \rightarrow 0$.

There are a variety of similar phase-field models which have been studied. Many of these may be written succinctly as

$$
T_{t}+\left(1-p^{2}\right)^{n} p_{t}=a^{2} \Delta T=0
$$

and

$$
\alpha \delta p_{t}-\lambda^{2} \delta^{2} \Delta p=\mu T\left(1-p^{2}\right)^{m}+p\left(1-p^{2}\right)
$$

where $n$ and $m$ are non-negative integers.

In [1-3] Caginalp focused primarily on the case where $n=m=0$. In [4] Caginalp and Chen considered (1.4) and (1.5) when $n=0$ and $m=2$. The case where $n=m=2$ has been studied by Sekerka and collaborators in $[7,11]$. The situation where $n=m=0$ is slightly different than when $n$ and $m$ are both $\geq 1$ because the latter situation loses the pointwise estimates $-1 \leq p(x, y, t) \leq 1$. Fabbri and Vollmer [6] have also noted this difference.

All of these models yield a diffuse interface description of melting and solidification. Corresponding to each particular model there is a corresponding sharp-interface description which is obtained by letting $\delta$ tend to $0^{+}$. For the system (1.1) and (1.2) the sharp-interface description takes the following simple form. $p$ takes on only the values -1 and +1 . Solid regions are those where $p=-1$ and liquid regions those where $p=+1$. In what follows

$$
\Gamma(t)=\{(x, y) \mid x=\hat{x}(s, t), y=\hat{y}(s, t), 0 \leq s \leq L\}
$$

will be a curve separating liquid and solid regions and $\boldsymbol{n}(s, t)$ will be the unit normal to $\Gamma$ which points into the liquid region. The unit tangent, $\boldsymbol{t}$, to $\Gamma$ is chosen so that $\boldsymbol{n}(s, t) \times \boldsymbol{t}(s, t)=\boldsymbol{e}_{3}$. $K$ is the curvature of $\Gamma$ at $(\hat{x}, \hat{y})(s, t)$, and

$$
c(s, t) \stackrel{\text { def }}{=}\left(\hat{x}_{t}, \hat{y}_{t}\right) \cdot \boldsymbol{n}(s, t)
$$

is the speed at which $\Gamma$ moves in the direction $\boldsymbol{n}$. A singular perturbation analysis of (1.1) and (1.2) yields the following results:

1. $T$ is continuous across $\Gamma$;

2. On $\Gamma$ the Gibbs-Thomson relation

$$
\alpha c+\lambda^{2} \delta K=-2^{1 / 2} \lambda \mu T_{M}
$$

holds; and finally across $\Gamma$ (1.1) implies that

$$
\frac{4}{3} c=a^{2}\left(\frac{\partial T^{-}}{\partial n}-\frac{\partial T^{+}}{\partial n}\right) .
$$

In (1.8), $T_{M}(s)=T(\hat{x}(s, t), \hat{y}(s, t))$, is the local melt temperature while in (1.9) $\frac{\partial T^{+}}{\partial n}$ is the normal derivative of $T$ in the liquid region and $\frac{\partial T^{-}}{\partial n}$ is the normal derivative of $T$ in the solid region. In the sharp-interface description one solves the heat equation

$$
T_{t}-a^{2} \Delta T=0
$$


in the portion of $\Omega$ away from interfaces. At equilibrium, $c=0$ and (1.8) implies that $T_{M}=O(\delta)$. In what follows, we shall restrict our attention to initial data $T\left(x, y, 0^{+}\right)$that is at worst $O\left(\delta^{\frac{1}{2}}\right)$.

The sharp-interface description becomes unduly complicated in the presence of multiple interfaces since all must be tracked. Difficulties also arise when interfaces merge. For these, and a plethora of other reasons, we focus on (1.1) and (1.2). Our principal results consist of a sequence of a priori inequalities satisfied by solutions of the system (1.1) and (1.2). These estimates guarantee regularity of the solutions and that the solutions converge to non-constant equilibria as $t$ tends to infinity.

One point worthy of note is that it is possible to obtain solutions of (1.1) and (1.2) which approximate those generated by the sharp interface descriptions. These are produced by solving (1.1) and (1.2) with initial data $p^{\delta}\left(x, y, 0^{+}\right)$which approximate the jump discontinuous data of the sharp interface theory but transition smoothly from -1 to +1 over an interval of width of $O(\delta)$ in the direction $\boldsymbol{n}$. For such initial $p$ 's (and initial temperature fields satisfying $\left.T\left(x, y, 0^{+}\right)=O\left(\delta^{\frac{1}{2}}\right)\right)$ we are guaranteed that terms like $\delta \iint_{\Omega}\left|\nabla p^{\delta}\right|^{2} \mathrm{~d} x \mathrm{~d} y$ and $\frac{1}{\delta} \iint_{\Omega}\left(1-p^{2}\right)^{2} \mathrm{~d} x \mathrm{~d} y$ are bounded independently of $\delta$ and $t$. These are the estimates which link the solutions of the two theories.

We note there is an "intermediate" model with the same equilibrium as the full system (1.1) and (1.2). In the "intermediate" model one replaces the local energy equation with the following identity for the average temperature:

$$
T_{0}(t)=h_{0}-\int_{-1 / 2}^{1 / 2} \int_{-1 / 2}^{1 / 2}\left(p-p^{3} / 3\right)(x, y, t) \mathrm{d} x \mathrm{~d} y
$$

and solves

$$
\alpha \delta p_{t}-\lambda^{2} \delta^{2} \Delta p=\left(\mu T_{0}+p\right)\left(1-p^{2}\right),(x, y) \in \Omega
$$

and

$$
\frac{\partial p}{\partial n}=0, \quad(x, y) \in \partial \Omega
$$

The constant $h_{0}$ in (1.10) is calculated from the initial data for the full system by

$$
h_{0}=\int_{-1 / 2}^{1 / 2} \int_{-1 / 2}^{1 / 2}\left(T+p-p^{3} / 3\right)\left(x, y, 0^{+}\right) \mathrm{d} x \mathrm{~d} y .
$$

This "intermediate" model is justified by two facts. The first is that solutions of (1.1) and (1.2) satisfying the boundary conditions $\frac{\partial T}{\partial n}=\frac{\partial p}{\partial n}=0,(x, y) \in \partial \Omega$ satisfy the overall balance equation

$$
h_{0} \equiv \int_{-1 / 2}^{1 / 2} \int_{-1 / 2}^{1 / 2}\left(T+p-p^{3} / 3\right)(x, y, t) \mathrm{d} x \mathrm{~d} y
$$

for all $t \geq 0$ and the second is that the spatially varying portion of $T$ decays to zero as $t$ tends to infinity (for details see the comments following (2.17)). The second observation allows us to replace the local temperature in (1.2) by the spatial average defined in (1.10). 


\section{Entropies AND A PRIORI ESTIMATES}

Our goal in this section is a set of inequalities of the form

$$
\frac{\partial \eta}{\partial t}-\operatorname{div} \boldsymbol{q} \leq G
$$

satisfied by solutions of

$$
\begin{array}{ll}
T_{t}+\left(1-p^{2}\right) p_{t}-a^{2} \Delta T=0, & (x, y) \in \Omega, \\
\alpha \delta p_{t}-\lambda^{2} \delta^{2} \Delta p=(\mu T+p)\left(1-p^{2}\right),(x, y) \in \Omega
\end{array}
$$

and

$$
\frac{\partial T}{\partial n}=\frac{\partial p}{\partial n}=0,(x, y) \in \partial \Omega
$$

Once again

$$
\Omega=\{(x, y) \mid-1 / 2<x<1 / 2 \text { and }-1 / 2<y<1 / 2)\}
$$

For (2.1) to be of value we require

$$
0 \leq \eta,(x, y) \in \Omega \text { and } \boldsymbol{q} \cdot \boldsymbol{n}=0,(x, y) \in \partial \Omega
$$

and that either $G \leq 0$ in $\Omega$ or $G$ is a priori bounded in $L_{1}(\Omega \times(0, \infty))$. Throughout we exploit the pointwise estimates

$$
-1 \leq p(x, y, t) \leq 1,(x, y) \in \Omega \text { and } t \geq 0
$$

satisfied by solutions of (2.3).

Once again we record the overall energy balance satisfied by solutions of $(2.2)-(2.4)$, namely the identity:

$$
\int_{-1 / 2}^{1 / 2} \int_{-1 / 2}^{1 / 2}\left(T+p-p^{3} / 3\right)(x, y, t) \mathrm{d} x \mathrm{~d} y \equiv h_{0}
$$

This is obtained by integrating (2.2) over $\Omega$ and exploiting $(2.4)_{1}$.

Our first inequality is obtained by multiplying $(2.2)$ by $\left(\frac{\mu T}{\delta}\right)$ and $(2.3)$ by $\frac{p_{t}}{\delta}$ and adding the resulting identities. This yields (2.1) with

$$
\begin{aligned}
& \eta_{1}=\frac{\mu T^{2}}{2 \delta}+\frac{\lambda^{2} \delta}{2}|\nabla p|^{2}+\frac{\left(1-p^{2}\right)^{2}}{4 \delta} \\
& \boldsymbol{q}_{1}=\frac{\mu a^{2}}{\delta} T \nabla T+\lambda^{2} \delta p_{t} \nabla p
\end{aligned}
$$

and

$$
G_{1}=-\alpha p_{t}^{2}-\frac{\mu a^{2}|\nabla T|^{2}}{\delta}
$$


and (2.8)-(2.10) yield

$$
\begin{aligned}
\frac{\mathrm{d}}{\mathrm{d} t} \int_{-1 / 2}^{1 / 2} \int_{-1 / 2}^{1 / 2}\left(\frac{\mu T^{2}}{2 \delta}+\frac{\lambda^{2} \delta}{2}|\nabla p|^{2}+\frac{\left(1-p^{2}\right)^{2}}{4 \delta}\right)(x, y, t) \mathrm{d} x \mathrm{~d} y & \\
& \leq-\int_{-1 / 2}^{1 / 2} \int_{-1 / 2}^{1 / 2}\left(\alpha p_{t}^{2}+\frac{\mu a^{2}}{\delta}|\nabla T|^{2}\right)(x, y, t) \mathrm{d} x \mathrm{~d} y .
\end{aligned}
$$

Once again we restrict our attention to initial data satisfying

$$
\int_{-1 / 2}^{1 / 2} \int_{-1 / 2}^{1 / 2}\left(\frac{T^{2}}{2 \delta}+\frac{\lambda^{2} \delta}{2}|\nabla p|^{2}+\frac{\left(1-p^{2}\right)^{2}}{4 \delta}\right)\left(x, y, 0^{+}\right) \mathrm{d} x \mathrm{~d} y=0(1)
$$

independent of $\delta$.

If we next multiply (2.2) by $\alpha T_{t}$ we obtain

$$
\frac{\alpha a^{2}}{2} \frac{\partial}{\partial t}|\nabla T|^{2}-\alpha a^{2} \operatorname{div}\left(T_{t} \nabla T\right)=-\alpha\left(T_{t}^{2}+\left(1-p^{2}\right) p_{t} T_{t}\right)
$$

and if we add this result to (2.1) with $\eta_{1}, \boldsymbol{q}_{1}, G_{1}$ we obtain

$$
\frac{\partial \eta_{2}}{\partial t}-\operatorname{div} \boldsymbol{q}_{2} \leq G_{2}
$$

where

$$
\begin{aligned}
& \eta_{2}=\frac{\mu T^{2}}{2 \delta}+\frac{\lambda^{2} \delta}{2}|\nabla p|^{2}+\frac{\left(1-p^{2}\right)^{2}}{4 \delta}+\frac{\alpha a^{2}}{2}|\nabla T|^{2} \\
& \boldsymbol{q}_{2}=\frac{\mu a^{2}}{\delta} T \nabla T+\lambda^{2} \delta p_{t} \nabla p+\alpha a^{2} T_{t} \nabla T
\end{aligned}
$$

and

$$
G_{2}=-\alpha\left(p_{t}^{2}+T_{t}^{2}+\left(1-p^{2}\right) T_{t} p_{t}\right)-\frac{\mu a^{2}}{\delta}|\nabla T|^{2} \leq \frac{-\alpha}{2}\left(p_{t}^{2}+T_{t}^{2}\right)-\frac{\mu a^{2}}{\delta}|\nabla T|^{2}
$$

and (2.12)-(2.14) imply that

$$
\begin{aligned}
\frac{\mathrm{d}}{\mathrm{d} t} \int_{-1 / 2}^{1 / 2} \int_{-1 / 2}^{1 / 2}\left(\frac{\mu T^{2}}{2 \delta}+\frac{\lambda^{2} \delta}{2}|\nabla p|^{2}+{\frac{\left(1-p^{2}\right)^{2}}{4 \delta}}^{2}\right. & \left.+\frac{\alpha a^{2}}{2}|\nabla T|^{2}\right)(x, y, t) \mathrm{d} x \mathrm{~d} y \\
\leq & -\int_{-1 / 2}^{1 / 2} \int_{-1 / 2}^{1 / 2}\left(\frac{\alpha}{2}\left(p_{t}^{2}+T_{t}^{2}\right)+\frac{\mu a^{2}}{\delta}|\nabla T|^{2}\right)(x, y, t) \mathrm{d} x \mathrm{~d} y .
\end{aligned}
$$

If we now write

$$
T(x, y, t)=T_{0}(t)+T_{1}(x, y, t), \quad(x, y) \in \Omega
$$

where

$$
T_{0}(t) \equiv \int_{-1 / 2}^{1 / 2} \int_{-1 / 2}^{1 / 2} T(x, y, t) \mathrm{d} x \mathrm{~d} y \text { and } \int_{-1 / 2}^{1 / 2} \int_{-1 / 2}^{1 / 2} T_{1}(x, y, t) \mathrm{d} x \mathrm{~d} y \equiv 0,
$$


then (2.15) implies that if $T$ and $p$ are in $H^{1}(\Omega)$ at $t=0$, then they are in $H^{1}(\Omega)$ for all $t>0$ and that $T$ satisfies $\lim _{t \rightarrow \infty} \int_{-1 / 2}^{1 / 2} \int_{-1 / 2}^{1 / 2} T_{1}^{2}(x, y, t) \mathrm{d} x \mathrm{~d} y=0$.

If we make a similar decomposition of $p$, that is write

$$
p(x, y, t)=p_{0}(t)+p_{1}(x, y, t)
$$

where

$$
p_{0}(t) \equiv \int_{-1 / 2}^{1 / 2} \int_{-1 / 2}^{1 / 2} p(x, y, t) \mathrm{d} x \mathrm{~d} y \text { and } \int_{-1 / 2}^{1 / 2} \int_{-1 / 2}^{1 / 2} p_{1}(x, y, t) \mathrm{d} x \mathrm{~d} y \equiv 0,
$$

then (2.3) implies that

$$
\alpha \delta \frac{\mathrm{d} p_{0}}{\mathrm{~d} t}=\int_{-1 / 2}^{1 / 2} \int_{-1 / 2}^{1 / 2}(\mu T+p)\left(1-p^{2}\right)(x, y, t) \mathrm{d} x \mathrm{~d} y
$$

and

$$
\alpha \delta \frac{\mathrm{d}^{2} p_{0}}{\mathrm{~d} t^{2}}=\int_{-1 / 2}^{1 / 2} \int_{-1 / 2}^{1 / 2}\left(\mu\left(T_{t}\left(1-p^{2}\right)-2 p T p_{t}\right)+\left(1-3 p^{2}\right) p_{t}\right)(x, y, t) \mathrm{d} x \mathrm{~d} y
$$

and (2.20) and (2.21) along with the fact that (2.15) implies that $p_{t}$ and $T_{t}$ are $L_{2}(\Omega \times(0, \infty))$ combine to yield the result that $\frac{\mathrm{d} p_{0}}{\mathrm{~d} t}$ and $\frac{\mathrm{d}^{2} p_{0}}{\mathrm{~d} t^{2}}$ are in $L_{2}(0, \infty)$ and that

$$
\lim _{t \rightarrow \infty} \alpha \delta \frac{\mathrm{d} p_{0}}{\mathrm{~d} t}=\lim _{t \rightarrow \infty} \int_{-1 / 2}^{1 / 2} \int_{-1 / 2}^{1 / 2}(\mu T+p)\left(1-p^{2}\right)(x, y, t) \mathrm{d} x \mathrm{~d} y=0 .
$$

With the estimate (2.15) we can derive weak information about the long-term behavior of solutions of (2.2)-(2.4). In what follows we let

$$
\left(T^{t}, p^{t}\right)(x, y, s)=(T, p)(x, y, t+s),(x, y) \in \Omega \text { and } 0 \leq s \leq s_{*}
$$

The inequality (2.15), together with (2.7), implies that for any $s_{*}$ the functions $T^{t}$ and $p^{t}$ are in $H^{1}\left(\Omega \times\left(0, s_{*}\right)\right)$ with bounds which depend only on the $H^{1}(\Omega)$ norms of $T\left(x, y, 0^{+}\right)$and $p\left(x, y, 0^{+}\right)$and $s_{*}$ and that

$$
\lim _{t \rightarrow \infty} \int_{0}^{s_{*}}\left(\int_{-1 / 2}^{1 / 2} \int_{-1 / 2}^{1 / 2}\left(\frac{\alpha}{2}\left(\left(T_{s}^{t}\right)^{2}+\left(p_{s}^{t}\right)^{2}\right)+\frac{\mu a^{2}}{\delta}\left|\nabla T^{t}\right|^{2}\right)(x, y, s) \mathrm{d} x \mathrm{~d} y\right) \mathrm{d} s=0 .
$$

Moreover, (2.3) implies that $\lambda^{2} \delta^{2} \Delta p^{t} \in L_{2}\left(\Omega \times\left(0, s_{*}\right)\right)$ with bounds which are independent of $t$. The boundedness of $T^{t}$ and $p^{t}$ in $H^{1}\left(\Omega \times\left(0, s_{*}\right)\right)$ implies we can find an increasing sequence $\left\{t_{i}\right\}_{i=1}^{\infty}$ with $\lim _{i \rightarrow \infty} t_{i}=\infty$ and functions $T^{\infty}$ and $p^{\infty}$ in $H^{1}\left(\Omega \times\left(0, s_{*}\right)\right)$ with $\Delta p^{\infty}$ in $L_{2}\left(\Omega \times\left(0, s_{*}\right)\right)$ with the following properties:

$\left(T^{\infty}, p^{\infty}\right)$ is the strong $L_{2}\left(\Omega \times\left(0, s_{*}\right)\right)$ limit of $\left(T^{t_{i}}, p^{t_{i}}\right)$ while $T_{s}^{\infty}, p_{s}^{\infty}, \nabla T^{\infty}, \nabla p^{\infty}$, and $\Delta p^{\infty}$ are the weak $L_{2}\left(\Omega \times\left(0, s_{*}\right)\right)$ limits of the appropriate derivatives of $T^{t_{i}}$ and $p^{t_{i}}$. Additionally, (2.24) implies

$$
T_{s}^{\infty}=p_{s}^{\infty}=0 \text { and } \nabla T^{\infty}=0 \text { in } \Omega \times\left(0, s_{*}\right)
$$


and thus that $T^{\infty}$ is a constant and $p^{\infty}$ is independent of $s$. The energy balance (1.13) yields

$$
T^{\infty}=h_{0}-\int_{-1 / 2}^{1 / 2} \int_{-1 / 2}^{1 / 2}\left(p^{\infty}-\left(p^{\infty}\right)^{3} / 3\right)(x, y) \mathrm{d} x \mathrm{~d} y
$$

while (2.7) implies that $-1 \leq p^{\infty} \leq 1$. (2.3) and (2.24) also imply that $p^{\infty}$ satisfies the equilibrium equation

$$
\lambda^{2} \delta^{2} \Delta p^{\infty}+\left(\mu T^{\infty}+p^{\infty}\right)\left(1-\left(p^{\infty}\right)^{2}\right)=0,(x, y) \in \Omega
$$

and

$$
\frac{\partial p^{\infty}}{\partial n}=0,(x, y) \in \partial \Omega
$$

where $T^{\infty}$ is given by (2.26). Moreover, (2.27) and (2.28) imply that $p^{\infty}$ is a critical point of the functional

$$
N\left(p^{\infty}\right) \stackrel{\text { def }}{=} \frac{\mu\left(T^{\infty}\right)^{2}}{2 \delta}+\int_{-1 / 2}^{1 / 2} \int_{-1 / 2}^{1 / 2}\left(\frac{\lambda^{2} \delta}{2}\left|\nabla p^{\infty}\right|^{2}+\frac{\left(1-\left(p^{\infty}\right)^{2}\right)^{2}}{4 \delta}\right)(x, y) \mathrm{d} x \mathrm{~d} y
$$

and that

$$
\eta\left(t, s_{*}\right) \stackrel{\text { def }}{=} \frac{1}{s_{*}} \int_{0}^{s_{*}} \int_{-1 / 2}^{1 / 2} \int_{-1 / 2}^{1 / 2}\left(\frac{\mu\left(T^{t}\right)^{2}}{2 \delta}+\frac{\lambda^{2} \delta}{2}\left|\nabla p^{t}\right|^{2}+\frac{\left(1-\left(p^{t}\right)^{2}\right)^{2}}{4 \delta}+\frac{\alpha a^{2}}{2}\left|\nabla T^{t}\right|^{2}\right)(x, y, s) \mathrm{d} x \mathrm{~d} y \mathrm{~d} s
$$

is decreasing in $t$ and has a limit $\eta_{\infty}$ satisfying

$$
N\left(p^{\infty}\right) \leq \eta_{\infty} \leq \eta\left(0^{+}\right)=O(1) .
$$

The last inequality guarantees that if the constant $h_{0}$ defined in (1.13) is small enough then $p^{\infty}$ is not identically constant. For simplicity we demonstrate this when $h_{0}=0$ and when the initial data for $T$ is the constant

$$
T\left(x, y, 0^{+}\right)=\iint_{\Omega}\left(p-p^{3} / 3\right)\left(x, y, 0^{+}\right) \mathrm{d} x \mathrm{~d} y .
$$

We also assume that $p\left(\cdot, \cdot, 0^{+}\right)$is such that $T\left(\cdot, \cdot, 0^{+}\right)=O\left(\delta^{1 / 2}\right)$ and that $\iint_{\Omega} \eta_{2}\left(x, y, 0^{+}\right) \mathrm{d} x \mathrm{~d} y=O(1)$ (see (2.12)). These constraints on the data guarantee that the limit $\eta_{\infty}$ in (2.30) is $O(1)$.

If we now suppose that $p^{\infty}$ is constant on $\Omega$, then $N\left(p^{\infty}\right)$ reduces to

$$
F\left(p^{\infty}\right)=\frac{\mu}{2 \delta}\left(p^{\infty}-\left(p^{\infty}\right)^{3} / 3\right)^{2}+\frac{\left(1-\left(p^{\infty}\right)^{2}\right)^{2}}{4 \delta}
$$

and $p^{\infty}$ is a critical point of $F$; i.e., satisfies

$$
\left(1-p^{2}\right)\left((\mu-1) p-\frac{\mu p^{3}}{3}\right)=0
$$

But, the assumption $0<\mu \leq 1$ guarantees the critical points are $(-1,0,1)$ and the associated critical values are $\left(\frac{2 \mu}{9 \delta}, \frac{1}{4 \delta}, \frac{2 \mu}{9 \delta}\right)$. For $0<\delta \ll 1$, all of these critical values are greater than $\eta_{\infty}$ and this, along with (2.31), confirms that the limits we obtain correspond to non-constant equilibria. 
To obtain stronger information we examine the time differentiated versions of (2.2)-(2.4), namely the equations:

$$
\begin{array}{ll}
T_{t t}+\left(1-p^{2}\right) p_{t t}-2 p p_{t}^{2}-a^{2} \Delta T_{t}=0, & (x, y) \in \Omega \\
\alpha \delta p_{t t}-\lambda^{2} \delta^{2} \Delta p_{t}=\left(\mu T_{t}+p_{t}\right)\left(1-p^{2}\right)-2 p(\mu T+p) p_{t}, & (x, y) \in \Omega \\
\frac{\partial T_{t}}{\partial n}=\frac{\partial p_{t}}{\partial n}=0, & (x, y) \in \partial \Omega
\end{array}
$$

We shall also exploit

Lemma 1. Suppose $f \in H^{1}(\Omega)$. Then, for every $\epsilon>0$

$$
\int_{-1 / 2}^{1 / 2} \int_{-1 / 2}^{1 / 2} f^{4}(x, y) \mathrm{d} x \mathrm{~d} y \leq 4\left[\frac{(1+\epsilon)}{\epsilon} \int_{-1 / 2}^{1 / 2} \int_{-1 / 2}^{1 / 2} f^{2}(x, y) \mathrm{d} x \mathrm{~d} y+\epsilon \int_{-1 / 2}^{1 / 2} \int_{-1 / 2}^{1 / 2}|\nabla f|^{2}(x, y) \mathrm{d} x \mathrm{~d} y\right]^{2} .
$$

We multiply (2.33) by $p_{t}$ and make use of (2.7) to obtain

$$
\frac{\alpha \delta}{2} \frac{\partial}{\partial t} p_{t}^{2}-\lambda^{2} \delta^{2} \operatorname{div}\left(p_{t} \nabla p_{t}\right) \leq-\lambda^{2} \delta^{2}\left|\nabla p_{t}\right|^{2}+\left(\frac{\mu T_{t}^{2}}{2}+\left(1+\frac{\mu}{2}\right) p_{t}^{2}\right)+2 \mu|T| p_{t}^{2}
$$

We note that $(2.15)$ implies the term $\frac{\mu T_{t}^{2}}{2}+\left(1+\frac{\mu}{2}\right) p_{t}^{2}$ is in $L_{1}(\Omega \times(0, \infty))$ and thus we confine our attention to $2 \mu|T| p_{t}^{2}$. Schwarz's inequality and Lemma 1 imply

$$
\begin{aligned}
& 2 \mu \int_{-1 / 2}^{1 / 2} \int_{-1 / 2}^{1 / 2}\left(|T| p_{t}^{2}\right)(x, y, t) \mathrm{d} x \mathrm{~d} y \leq 2 \mu\left(\int_{-1 / 2}^{1 / 2} \int_{-1 / 2}^{1 / 2} T^{2}(x, y, t) \mathrm{d} x \mathrm{~d} y\right)^{1 / 2}\left(\int_{-1 / 2}^{1 / 2} \int_{-1 / 2}^{1 / 2} p_{t}^{4}(x, y, t) \mathrm{d} x \mathrm{~d} y\right)^{1 / 2} \\
& \leq 2 \mu\left(\int_{-1 / 2}^{1 / 2} \int_{-1 / 2}^{1 / 2} T^{2}(x, y, t) \mathrm{d} x \mathrm{~d} y\right)^{1 / 2}\left(\left(\frac{1+\epsilon}{\epsilon}\right) \int_{-1 / 2}^{1 / 2} \int_{-1 / 2}^{1 / 2} p_{t}^{2}(x, y, t) \mathrm{d} x \mathrm{~d} y+\left.\epsilon \int_{-1 / 2}^{1 / 2} \int_{-1 / 2}^{1 / 2} \nabla p_{t}\right|^{2}(x, y, t) \mathrm{d} x \mathrm{~d} y\right) .
\end{aligned}
$$

Moreover, (2.11) guarantees that

$$
\left(\int_{-1 / 2}^{1 / 2} \int_{-1 / 2}^{1 / 2} T^{2}(x, y, t) \mathrm{d} x \mathrm{~d} y\right)^{1 / 2} \leq\left(\frac{2 \delta}{\mu} \int_{-1 / 2}^{1 / 2} \int_{-1 / 2}^{1 / 2} \eta_{1}\left(x, y, 0^{+}\right) \mathrm{d} x \mathrm{~d} y\right)^{1 / 2}
$$

and this guarantees that we may choose $\epsilon$ so that

$$
2 \mu \epsilon\left(\int_{-1 / 2}^{1 / 2} \int_{-1 / 2}^{1 / 2} T^{2}(x, y, t) \mathrm{d} x \mathrm{~d} y\right)^{1 / 2} \int_{-1 / 2}^{1 / 2} \int_{-1 / 2}^{1 / 2}\left|\nabla p_{t}\right|^{2}(x, y, t) \leq \frac{\lambda^{2} \delta^{2}}{2} \int_{-1 / 2}^{1 / 2} \int_{-1 / 2}^{1 / 2}\left|\nabla p_{t}\right|^{2}(x, y, t) \mathrm{d} x \mathrm{~d} y
$$


With $\epsilon$ so chosen, (2.35) implies that

$$
\begin{aligned}
& \frac{\alpha \delta}{2} \frac{\mathrm{d}}{\mathrm{d} t} \int_{-1 / 2}^{1 / 2} \int_{-1 / 2}^{1 / 2} p_{t}^{2}(x, y, t) \leq \frac{-\lambda^{2} \delta^{2}}{2} \int_{-1 / 2}^{1 / 2} \int_{-1 / 2}^{1 / 2}\left|\nabla p_{t}\right|^{2}(x, y, t) \mathrm{d} x \mathrm{~d} y+\frac{\mu}{2} \int_{-1 / 2}^{1 / 2} \int_{-1 / 2}^{1 / 2} T_{t}^{2}(x, y, t) \mathrm{d} x \mathrm{~d} y \\
& +\left(1+\frac{\mu}{2}+2^{3 / 2}\left(\mu \delta \int_{-1 / 2}^{1 / 2} \int_{-1 / 2}^{1 / 2} \eta_{1}\left(x, y, 0^{+}\right) \mathrm{d} x \mathrm{~d} y\right)^{1 / 2}\left(1+\frac{1}{\epsilon}\right)\right) \int_{-1 / 2}^{1 / 2} \int_{-1 / 2}^{1 / 2} p_{t}^{2}(x, y, t) \mathrm{d} x \mathrm{~d} y
\end{aligned}
$$

and (2.39) then implies that if

$$
p_{t}\left(x, y, 0^{+}\right)=\frac{1}{\alpha \delta}\left(\lambda^{2} \delta^{2} \Delta p+(\mu T+p)\left(1-p^{2}\right)\right)\left(x, y, 0^{+}\right)
$$

is in $L_{2}(\Omega)$, then $p_{t}$ and thus $\Delta p$ are in $L_{2}(\Omega)$ independently of $t$ and $\nabla p_{t} \in L_{2}(\Omega \times(0, \infty))$.

The preceding estimate points up the advantage of the "intermediate" model discussed briefly in (1.9)-(1.12) over the full system. For the intermediate model the cantankerous term $2 \mu|T| p_{t}^{2}$ in (2.35) would have been replaced by $2 \mu\left|T_{0}\right| p_{t}^{2}$ and this latter term is easily controlled since we know by our previous estimates that

$$
\left|T_{0}\right| \leq\left(\frac{2 \delta}{\mu} \int_{-1 / 2}^{1 / 2} \int_{-1 / 2}^{1 / 2} \eta_{1}\left(x, y, 0^{+}\right) \mathrm{d} x \mathrm{~d} y\right)^{1 / 2}
$$

and thus we would not have to invoke Lemma 1 and choose $\epsilon=0\left(\delta^{3 / 2}\right)$. This latter choice of $\epsilon$ pumped up the coefficient of $\int_{-1 / 2}^{1 / 2} \int_{-1 / 2}^{1 / 2} p_{t}^{2}(x, y, t) \mathrm{d} x \mathrm{~d} y$ in $(2.39)$ to be $O\left(1 / \delta^{3 / 2}\right)$ which is far from optimal. If we chose to add some multiple of (2.39) to (2.15) and wanted the right hand side of the resulting inequality to be negative definite our multiplier would have to be $O\left(\delta^{3 / 2}\right)$, an unacceptably small number. For the "intermediate" model we could use an $O(1)$ multiplier (for details see $(2.61)-(2.63)$ ).

On the other hand, if we multiply $(2.32)$ by $\left(T_{t}+\left(1-p^{2}\right) p_{t}\right) / a^{2}$ we find that

$$
\begin{aligned}
& \frac{1}{2 a^{2}} \frac{\partial}{\partial t}\left(T_{t}+\left(1-p^{2}\right) p_{t}\right)^{2}=\frac{a^{2}}{2} \frac{\partial}{\partial t}(\Delta T)^{2} \\
& =\operatorname{div}\left(\left(T_{t}+\left(1-p^{2}\right) p_{t}\right) \nabla T_{t}\right)-\left|\nabla T_{t}\right|^{2}-\left(1-p^{2}\right) \nabla T_{t} \cdot \nabla p_{t}+2 p p_{t} \nabla T_{t} \cdot \nabla p
\end{aligned}
$$

and (2.40) implies that

$$
\begin{aligned}
& \frac{a^{2}}{2} \frac{\mathrm{d}}{\mathrm{d} t} \int_{-1 / 2}^{1 / 2} \int_{-1 / 2}^{1 / 2}(\Delta T)^{2}(x, y, t) \mathrm{d} x \mathrm{~d} y \leq \\
& -\frac{1}{2} \int_{-1 / 2}^{1 / 2} \int_{-1 / 2}^{1 / 2}\left|\nabla T_{t}\right|^{2}(x, y, t) \mathrm{d} x \mathrm{~d} y+\int_{-1 / 2}^{1 / 2} \int_{-1 / 2}^{1 / 2}\left|\nabla p_{t}\right|^{2}(x, y, t) \mathrm{d} x \mathrm{~d} y \\
& +4 \int_{-1 / 2}^{1 / 2} \int_{-1 / 2}^{1 / 2} p_{t}^{2}|\nabla p|^{2}(x, y, t) \mathrm{d} x \mathrm{~d} y
\end{aligned}
$$

The first term on the right hand side of (2.41) is in controllable by virtue of the minus sign and the second term is $L_{1}(0, \infty)$ by virtue of $(2.39)$. Thus we can confine our attention to the third term. To show this term 
is $L_{1}(0, \infty)$ we note that

$$
\int_{-1 / 2}^{1 / 2} \int_{-1 / 2}^{1 / 2} p_{t}^{2}|\nabla p|^{2}(x, y, t) \mathrm{d} x \mathrm{~d} y \leq\left(\int_{-1 / 2}^{1 / 2} \int_{-1 / 2}^{1 / 2} p_{t}^{4}(x, y, t) \mathrm{d} x \mathrm{~d} y\right)^{1 / 2}\left(\int_{-1 / 2}^{1 / 2} \int_{-1 / 2}^{1 / 2}|\nabla p|^{4}(x, y, t) \mathrm{d} x \mathrm{~d} y\right)^{1 / 2} .
$$

Lemma 1 then guarantees that

$$
\left(\int_{-1 / 2}^{1 / 2} \int_{-1 / 2}^{1 / 2} p_{t}^{4}(x, y, t) \mathrm{d} x \mathrm{~d} y\right)^{1 / 2} \leq(1+\sqrt{5})\left(\int_{-1 / 2}^{1 / 2} \int_{-1 / 2}^{1 / 2}\left(p_{t}^{2}+\left|\nabla p_{t}\right|^{2}\right)(x, y, t) \mathrm{d} x \mathrm{~d} y\right)
$$

and that

$$
\begin{aligned}
& \left(\int_{-1 / 2}^{1 / 2} \int_{-1 / 2}^{1 / 2}|\nabla p|^{4}(x, y, t) \mathrm{d} x \mathrm{~d} y\right)^{1 / 2} \\
\leq & 2^{1 / 2}\left(\int_{-1 / 2}^{1 / 2} \int_{-1 / 2}^{1 / 2} p_{x}^{4}(x, y, t) \mathrm{d} x \mathrm{~d} y\right)^{1 / 2}+2^{1 / 2}\left(\int_{-1 / 2}^{1 / 2} \int_{-1 / 2}^{1 / 2} p_{y}^{4}(x, y, t) \mathrm{d} x \mathrm{~d} y\right)^{1 / 2} \\
\leq & 2^{1 / 2}(1+\sqrt{5}) \int_{-1 / 2}^{1 / 2} \int_{-1 / 2}^{1 / 2}\left(p_{x}^{2}+p_{y}^{2}+p_{x x}^{2}+p_{y y}^{2}+2 p_{x y}^{2}\right)(x, y, t) \mathrm{d} x \mathrm{~d} y \\
\leq & 2^{1 / 2}(1+\sqrt{5}) \int_{-1 / 2}^{1 / 2} \int_{-1 / 2}^{1 / 2}\left(p_{x}^{2}+p_{y}^{2}+(\Delta p)^{2}\right)(x, y, t) \mathrm{d} x \mathrm{~d} y
\end{aligned}
$$

and (2.43) and (2.44) together with (2.41) guarantee that if $T\left(x, y, 0^{+}\right)$and $p\left(x, y, 0^{+}\right)$are in $H^{2}(\Omega)$ then $T(x, y, t)$ is in $H^{2}(\Omega)$ for all future times and additionally that $\nabla T_{t} \in L_{2}(\Omega \times(0, \infty))$.

The estimates (2.39) and (2.44) also guarantee that if $T\left(x, y, 0^{+}\right)$and $p\left(x, y, 0^{+}\right)$are in $H^{2}(\Omega)$, then the resulting orbit is compact in $H^{1}(\Omega)$ and that the $w$-limit set is nonempty in $H^{1}(\Omega)$. The fact that $\nabla T_{t} \in$ $L_{2}(\Omega \times(0, \infty))$ further guarantees that the function $T_{1}$ defined in $(2.16)$ and $(2.17)$ converges to zero strongly in $H^{1}(\Omega)$.

Our final estimates pertain to solutions generated by initial data $T\left(x, y, 0^{+}\right) \in H^{2}(\Omega)$ and $p\left(x, y, 0^{+}\right) \in H^{3}(\Omega)$. These are obtained by multiplying $(2.33)$ by $p_{t t}$. The resulting inequality is

$$
\begin{aligned}
& \frac{\lambda^{2} \delta^{2}}{2} \frac{\partial}{\partial t}\left|\nabla p_{t}\right|^{2}+\alpha \delta p_{t t}^{2}-\lambda^{2} \delta^{2} \operatorname{div}\left(p_{t t} \nabla p_{t}\right)= \\
& \mu\left(T_{t}\left(1-p^{2}\right)-2 p T p_{t}\right) p_{t t}+\left(1-3 p^{2}\right) p_{t} p_{t t} \\
& \leq \frac{\alpha \delta}{2} p_{t t}^{2}+\frac{2 \mu^{2}}{\alpha \delta}\left(T_{t}^{2}+4 T^{2} p_{t}^{2}\right)+\frac{8}{\alpha \delta} p_{t}^{2}
\end{aligned}
$$

Our preceding calculations imply that if $T\left(x, y, 0^{+}\right) \in H^{2}(\Omega)$ and $p\left(x, y, 0^{+}\right) \in H^{3}(\Omega)$, then

$$
\frac{2 \mu^{2}}{\alpha \delta}\left(T_{t}^{2}+4 T^{2} p_{t}^{2}\right)+\frac{8}{\alpha \delta} p_{t}^{2}
$$

is in $L_{1}(\Omega \times(0, \infty))$ and thus $(2.45)$ implies that $\nabla p_{t} \in L_{2}(\Omega)$ independently of $t$ and that $p_{t t} \in L_{2}(\Omega \times(0, \infty))$. The former conclusion, together with (2.3), implies that $p \in H^{3}(\Omega)$ with bounds which are independent of $t$ 
while the latter conclusion implies that

$$
\lim _{t \rightarrow \infty} \alpha \delta p_{t}=\lim _{t \rightarrow \infty}\left(\lambda^{2} \delta^{2} \Delta p+(\mu T+p)\left(1-p^{2}\right)\right)=0
$$

and that this limit is obtained strongly in $L_{2}(\Omega)$.

We conclude this section with some observations about the "intermediate" model introduced in (1.9)-(1.12). Once again in that model one replaces (2.3) with

$$
\alpha \delta p_{t}-\lambda^{2} \delta^{2} \Delta p=\left(\mu T_{0}+p\right)\left(1-p^{2}\right),(x, y) \in \Omega
$$

and

$$
\frac{\partial p}{\partial n}=0 \quad, \quad(x, y) \in \partial \Omega
$$

where

$$
T_{0}(t)=h_{0}-\int_{-1 / 2}^{1 / 2} \int_{-1 / 2}^{1 / 2}\left(p-p^{3} / 3\right)(x, y, t) \mathrm{d} x \mathrm{~d} y \text { and } h_{0}=\int_{-1 / 2}^{1 / 2} \int_{-1 / 2}^{1 / 2}\left(T+p-p^{3} / 3\right)\left(x, y, 0^{+}\right) \mathrm{d} x \mathrm{~d} y .
$$

Solutions of this latter system also satisfy the pointwise estimates $-1 \leq p(x, y, t) \leq 1$ and a set of "entropy" inequalities similar to those satisfied by solutions of the full system. The most basic of these is

$$
\frac{\partial \tilde{\eta}_{1}}{\partial t}-\operatorname{div} \tilde{\boldsymbol{q}}_{1}=\tilde{G}_{1}
$$

where

$$
\begin{aligned}
& \tilde{\eta}_{1}=\frac{\mu T_{0}^{2}}{2 \delta}+\frac{\lambda^{2} \delta}{2}|\nabla p|^{2}+\frac{\left(1-p^{2}\right)^{2}}{4 \delta} \\
& \tilde{\boldsymbol{q}}_{1}=\lambda^{2} \delta p_{t} \nabla p
\end{aligned}
$$

and

$$
\tilde{G}_{1}=-\alpha p_{t}^{2}
$$

Moreover, (2.49)-(2.52) imply that

$$
\frac{\mathrm{d}}{\mathrm{d} t} \int_{-1 / 2}^{1 / 2} \int_{-1 / 2}^{1 / 2}\left(\frac{\mu T_{0}^{2}}{2 \delta}+\frac{\lambda^{2} \delta}{2}|\nabla p|^{2}+\frac{\left(1-p^{2}\right)^{2}}{4 \delta}\right)(x, y, t) \mathrm{d} x \mathrm{~d} y \leq-\alpha \int_{-1 / 2}^{1 / 2} \int_{-1 / 2}^{1 / 2} p_{t}^{2}(x, y, t) \mathrm{d} x \mathrm{~d} y .
$$

The time differentiated identities

$$
\begin{gathered}
\alpha \delta p_{t t}-\lambda^{2} \delta^{2} \Delta p_{t}=\mu\left(\dot{T}_{0}\left(1-p^{2}\right)-2 p T_{0} p_{t}\right)+\left(1-3 p^{2}\right) p_{t}, \\
\dot{T}_{0}=-\int_{-1 / 2}^{1 / 2} \int_{-1 / 2}^{1 / 2}\left(1-p^{2}\right) p_{t}(x, y, t) \mathrm{d} x \mathrm{~d} y
\end{gathered}
$$

and

$$
\frac{\partial p_{t}}{\partial n}=0 \quad, \quad(x, y) \in \partial \Omega
$$


also yield:

$$
\begin{aligned}
& \frac{\alpha \delta}{2} \frac{\partial p_{t}^{2}}{\partial t}-\lambda^{2} \delta^{2} \operatorname{div}\left(p_{t} \nabla p_{t}\right)= \\
& -\lambda^{2} \delta^{2}\left|\nabla p_{t}\right|^{2}+\mu\left(\dot{T}_{0}\left(1-p^{2}\right)-2 p T_{0} p_{t}\right) p_{t}+\left(1-3 p^{2}\right) p_{t}^{2} \\
& \leq-\lambda^{2} \delta^{2}\left|\nabla p_{t}\right|^{2}+\frac{\mu}{2}\left(\int_{-1 / 2}^{1 / 2} \int_{-1 / 2}^{1 / 2} p_{t}^{2}(x, y, t) \mathrm{d} x \mathrm{~d} y\right) \\
& +2^{3 / 2} \mu^{1 / 2} \delta^{1 / 2}\left(\int_{-1 / 2}^{1 / 2} \int_{-1 / 2}^{1 / 2} \tilde{\eta}_{1}\left(x, y, 0^{+}\right) \mathrm{d} x \mathrm{~d} y\right)^{1 / 2} p_{t}^{2}+\left(1+\frac{\mu}{2}\right) p_{t}^{2}
\end{aligned}
$$

and

$$
\begin{aligned}
& \frac{\lambda^{2} \delta^{2}}{2} \frac{\partial\left|\nabla p_{t}\right|^{2}}{\partial t}-\lambda^{2} \delta^{2} \operatorname{div}\left(p_{t t} \nabla p_{t}\right)= \\
& -\alpha \delta p_{t t}^{2}+\mu\left(\dot{T}_{0}\left(1-p^{2}\right)-2 p T_{0} p_{t}\right) p_{t t}+\left(1-3 p^{2}\right) p_{t} p_{t t} \\
& \leq-\frac{\alpha \delta}{2} p_{t t}^{2}+\frac{1}{\alpha \delta}\left(2 \mu^{2} \int_{-1 / 2}^{1 / 2} \int_{-1 / 2}^{1 / 2} p_{t}^{2}(x, y, t) \mathrm{d} x \mathrm{~d} y+\left(4+16 \mu \delta \int_{-1 / 2}^{1 / 2} \int_{-1 / 2}^{1 / 2} \tilde{\eta}_{1}\left(x, y, 0^{+}\right) \mathrm{d} x \mathrm{~d} y\right) p_{t}^{2}\right) .
\end{aligned}
$$

The former follows from multiplying $(2.54)$ by $p_{t}$ and the latter from multiplying (2.54) by $p_{t t}$. Moreover, if we let

$$
\begin{aligned}
\beta_{2}= & \left(1+\mu+2^{3 / 2} \mu^{1 / 2} \delta^{1 / 2} \int_{-1 / 2}^{1 / 2} \int_{-1 / 2}^{1 / 2} \tilde{\eta}_{1}\left(x, y, 0^{+}\right) \mathrm{d} x \mathrm{~d} y\right), \\
\beta_{3}= & 4+2 \mu^{2}+16 \mu \delta \int_{-1 / 2}^{1 / 2} \int_{-1 / 2}^{1 / 2} \tilde{\eta}_{1}\left(x, y, 0^{+}\right) \mathrm{d} x \mathrm{~d} y, \\
\tilde{\eta}_{2}= & \tilde{\eta}_{1}+\frac{\alpha^{2} \delta}{8 \beta_{2}} p_{t}^{2}=\frac{\mu T_{0}^{2}}{2 \delta}+\frac{\lambda^{2} \delta}{2}|\nabla p|^{2}+\frac{\left(1-p^{2}\right)^{2}}{4 \delta}+\frac{\alpha^{2} \delta}{8 \beta_{2}} p_{t}^{2}, \\
\tilde{\boldsymbol{q}}_{2}= & \tilde{\boldsymbol{q}}_{1}+\frac{\alpha \lambda^{2} \delta^{2}}{4 \beta_{2}} p_{t} \nabla p_{t}=\lambda^{2} \delta p_{t} \nabla p+\frac{\alpha \lambda^{2} \delta^{2}}{4 \beta_{2}} p_{t} \nabla p_{t}, \\
\tilde{G}_{2}= & \tilde{G}_{1}+\frac{\alpha}{4 \beta_{2}}\left(1+\frac{\mu}{2}+2^{3 / 2} \mu^{1 / 2} \delta^{1 / 2} \int_{-1 / 2}^{1 / 2} \int_{-1 / 2}^{1 / 2} \tilde{\eta}_{1}\left(x, y, 0^{+}\right) \mathrm{d} x \mathrm{~d} y\right) p_{t}^{2} \\
& +\frac{\alpha \mu}{8 \beta_{2}} \int_{-1 / 2}^{1 / 2} \int_{-1 / 2}^{1 / 2} p_{t}^{2}(x, y, t) \mathrm{d} x \mathrm{~d} y-\frac{\alpha \lambda^{2} \delta^{2}}{4 \beta_{2}}\left|\nabla p_{t}\right|^{2}, \\
\tilde{\eta}_{3}= & \tilde{\eta}_{2}+\frac{\alpha^{2} \lambda^{2} \delta^{3}}{8 \beta_{3}}\left|\nabla p_{t}\right|^{2}=\frac{\mu T_{0}^{2}}{2 \delta}+\frac{\lambda^{2} \delta}{2}|\nabla p|^{2}+\frac{\left(1-p^{2}\right)}{4 \delta}+\frac{\alpha^{2} \delta}{8 \beta_{2}} p_{t}^{2}+\frac{\alpha^{2} \lambda^{2} \delta^{3}}{8 \beta_{3}}\left|\nabla p_{t}^{2}\right| \\
\tilde{\boldsymbol{q}}_{3}= & \tilde{\boldsymbol{q}}_{2}+\frac{\alpha^{2} \lambda^{2} \delta^{3}}{4 \beta_{3}} p_{t t} \nabla p_{t}=\lambda^{2} \delta p_{t} \nabla p+\frac{\alpha \lambda^{2} \delta^{2}}{4 \beta_{2}} p_{t} \nabla p_{t}+\frac{\alpha^{2} \lambda^{2} \delta^{3}}{4 \beta_{3}} p_{t t} \nabla p_{t},
\end{aligned}
$$


and

$$
\begin{aligned}
\tilde{G}_{3}= & \tilde{G}_{2}+\frac{\alpha}{4 \beta_{3}}\left(4+16 \mu \delta \int_{-1 / 2}^{1 / 2} \int_{-1 / 2}^{1 / 2} \tilde{\eta}_{1}\left(x, y, 0^{+}\right) \mathrm{d} x \mathrm{~d} y\right) p_{t}^{2} \\
& +\frac{\alpha}{2 \beta_{3}} \mu^{2} \int_{-1 / 2}^{1 / 2} \int_{-1 / 2}^{1 / 2} p_{t}^{2}(x, y, t) \mathrm{d} x \mathrm{~d} y-\frac{\alpha^{2} \delta^{2}}{8 \beta_{3}} p_{t t}^{2}
\end{aligned}
$$

we obtain the inequalities

$$
\frac{\partial \tilde{\eta}_{2}}{\partial t}-\operatorname{div} \tilde{\boldsymbol{q}}_{2} \leq \tilde{G}_{2} \text { and } \frac{\partial \tilde{\eta}_{3}}{\partial t}=\operatorname{div} \tilde{\boldsymbol{q}}_{3} \leq \tilde{G}_{3}
$$

and these imply

$$
\begin{aligned}
& \frac{\mathrm{d}}{\mathrm{d} t} \int_{-1 / 2}^{1 / 2} \int_{-1 / 2}^{1 / 2}\left(\frac{\mu T_{0}^{2}}{2 \delta}+\frac{\lambda^{2} \delta|\nabla p|^{2}}{2}+\frac{\left(1-p^{2}\right)^{2}}{4 \delta}+\frac{\alpha^{2} \delta}{8 \beta_{2}} p_{t}^{2}\right)(x, y, t) \mathrm{d} x \mathrm{~d} y \\
& \leq-\frac{3 \alpha}{4} \int_{-1 / 2}^{1 / 2} \int_{-1 / 2}^{1 / 2} p_{t}^{2}(x, y, t) \mathrm{d} x \mathrm{~d} y-\frac{\alpha \lambda^{2} \delta^{2}}{4 \beta_{2}} \int_{-1 / 2}^{1 / 2} \int_{-1 / 2}^{1 / 2}\left|\nabla p_{t}\right|^{2}(x, y, t) \mathrm{d} x \mathrm{~d} y
\end{aligned}
$$

and

$$
\begin{aligned}
\frac{\mathrm{d}}{\mathrm{d} t} \int_{-1 / 2}^{1 / 2} \int_{-1 / 2}^{1 / 2}\left(\frac{\mu T_{0}^{2}}{2 \delta}+\right. & \left.\frac{\lambda^{2} \delta|\nabla p|^{2}}{2}+\frac{\left(1-p^{2}\right)^{2}}{4 \delta}+\frac{\alpha \delta}{8 \beta_{2}} p_{t}^{2}+\frac{\alpha^{2} \lambda^{2} \delta^{3}}{8 \beta_{3}}\left|\nabla p_{t}\right|^{2}\right)(x, y, t) \mathrm{d} x \mathrm{~d} y \\
\leq-\frac{\alpha}{2} \int_{-1 / 2}^{1 / 2} \int_{-1 / 2}^{1 / 2} p_{t}^{2}(x, y, t)-\frac{\alpha \lambda^{2} \delta^{2}}{4 \beta_{2}} \int_{-1 / 2}^{1 / 2} \int_{-1 / 2}^{1 / 2}\left|\nabla p_{t}\right|^{2}(x, y, t) \mathrm{d} x \mathrm{~d} y & -\frac{\alpha^{2} \delta^{2}}{4 \beta_{3}} \int_{-1 / 2}^{1 / 2} \int_{-1 / 2}^{1 / 2} p_{t t}^{2}(x, y, t) \mathrm{d} x \mathrm{~d} y .
\end{aligned}
$$

We leave it to the reader to supply the precise conclusions derivable from the inequalities (2.53), (2.63), and (2.69). The key point of these inequalities is that the parameter $\delta$ appears optimally.

\section{Computational experiments}

In this section we present some numerical simulations of the system (2.2)-(2.4). We let $N$ be an integer and set

$$
\mathrm{d} x=\mathrm{d} y=1 / N .
$$

Our approximate solutions will be constant on the cells

$$
\Omega_{i, j}=\left\{(x, y) \mid-\frac{1}{2}+\frac{(i-1)}{N}<x<-\frac{1}{2}+\frac{i}{N} \text { and }-\frac{1}{2}+\frac{(j-1)}{N}<y<-\frac{1}{2}+\frac{j}{N}\right\}
$$

where $1 \leq i, j \leq N$ and thus the cell averages $\left(T_{i, j}, p_{i, j}\right)$ may also be thought of as the point values at the cell centers

$$
\left(x_{i}, y_{j}\right)=\left(-\frac{1}{2}+\frac{2 i-1}{2 N},-\frac{1}{2}+\frac{2 j-1}{2 N}\right)
$$


We impose the boundary conditions

$$
\frac{\partial T}{\partial n}=\frac{\partial p}{\partial n}=0, \quad(x, y) \in \partial \Omega
$$

by insisting that the extended grid functions satisfy

$$
\left.\begin{array}{lll} 
& & \left(T_{0, k}, p_{0, k}\right)=\left(T_{1, k}, p_{1, k}\right), \\
& \left(T_{N+1, k}, p_{N+1, k}\right)=\left(T_{N, k}, p_{N, k}\right), \\
& \left(T_{k, 0}, p_{k, 0}\right)=\left(T_{k, 1}, p_{k, 1}\right), \\
\text { and } & \\
& & \\
& \left(T_{k, N+1}, p_{k, N+1}\right)=\left(T_{k, N}, p_{k, N}\right)
\end{array}\right\}
$$

for $1 \leq k \leq N$.

For any function $u$ defined on the grid and satisfying (3.5) we let

$$
\Delta_{N} u_{i, j}=N^{2}\left(u_{i+1, j}+u_{i-1, j}+u_{i, j+1}+u_{i, j-1}-4 u_{i, j}\right)
$$

be the discrete Laplacian applied to $u$. For functions $u$ and $v$ satisfying (3.5) we define

$$
[u, v]=\sum_{i, j=1}^{N} u_{i, j} v_{i, j} / N^{2}
$$

and note that

$$
-\left[u, \Delta_{N} u\right]=\sum_{i=1}^{N} \sum_{j=1}^{N-1}\left(u_{i, j+1}-u_{i, j}\right)^{2}+\sum_{j=1}^{N} \sum_{i=1}^{N-1}\left(u_{i+1, j}-u_{i, j}\right)^{2}
$$

Such grid functions also satisfy the discrete version of Lemma 1, namely the inequality

$$
\left[u^{2}, u^{2}\right]=\left[u^{4}, 1\right] \leq 4\left(\frac{(1+\epsilon)}{\epsilon}[u, u]-\epsilon\left[u, \Delta_{N} u\right]\right)^{2}
$$

for every $\epsilon>0$.

Finally, we replace $(2.2)-(2.4)$ by the system

$$
\dot{T}_{i, j}+\left(1-p_{i, j}^{2}\right) \dot{p}_{i, j}-a^{2} \Delta_{N} T_{i, j}=0
$$

and

$$
\alpha \delta \dot{p}_{i, j}-\lambda^{2} \delta^{2} \Delta_{N} p_{i, j}=\left(\mu T_{i, j}+p_{i, j}\right)\left(1-p_{i, j}^{2}\right)
$$

where $T_{i, j}$ and $p_{i, j}$ satisfy the discrete boundary conditions (3.5) and the symbol $\cdot$ denotes differentiation with respect to $t$. Solutions of (3.10) and (3.11) obey the pointwise estimate that if $-1 \leq p_{i, j}\left(0^{+}\right) \leq 1,1 \leq i, j \leq N$, then $-1 \leq p_{i, j}(t) \leq 1,1 \leq i, j \leq N$ for all $t>0$. This system also satisfies estimates which are the exact analogue of those obtained in Section 2. Corresponding to (2.8) we have the identity

$$
\left[T+p-p^{3} / 3,1\right](t) \equiv h_{0}
$$


while the analogues of (2.8) and (2.15) are the inequalities

$$
\frac{\mathrm{d}}{\mathrm{d} t}\left(\left[\left(\frac{\mu T^{2}}{2 \delta}+\frac{\left(1-p^{2}\right)^{2}}{4 \delta}\right), 1\right]-\frac{\lambda^{2} \delta}{2}\left[p, \Delta_{N} p\right]\right)(t)=-\alpha\left[p_{t}, p_{t}\right](t)+\frac{\mu a^{2}}{\delta}\left[T, \Delta_{N} T\right](t),
$$

and

$$
\begin{aligned}
\frac{\mathrm{d}}{\mathrm{d} t}\left(\left[\frac{\mu T^{2}}{2 \delta}+\frac{\left(1-p^{2}\right)^{2}}{4 \delta}, 1\right]-\frac{\lambda^{2} \delta}{2}\left[p, \Delta_{N} p\right]-\frac{\alpha a^{2}}{2}\left[T, \Delta_{N} T\right]\right) & (t) \\
& \leq-\frac{\alpha}{2}\left(\left[p_{t}, p_{t}\right]+\left[T_{t}, T_{t}\right]\right)(t)+\frac{\mu a^{2}}{\delta}\left[T, \Delta_{N} T\right](t) .
\end{aligned}
$$

We spare the reader the detailed analogues of (2.39), (2.41), and (2.45). Suffice it to say if we replace terms like $\int_{-1 / 2}^{1 / 2} \int_{-1 / 2}^{1 / 2} f(x, y, t) \mathrm{d} x \mathrm{~d} y$ by $[f, 1](t)$ and terms like $\int_{-1 / 2}^{1 / 2} \int_{-1 / 2}^{1 / 2}|\nabla f|^{2}(x, y, t) \mathrm{d} x \mathrm{~d} y$ by $-\left[f, \Delta_{N} f\right](t)$, then the resulting inequalities go through intact.

When doing simulations with the discrete system (3.10) and (3.11) we link the small parameter $\delta$ to the grid $\mathrm{d} x=\mathrm{d} y=1 / N ;$ specifically we set

$$
\delta=1 / N
$$

and restrict our attention to initial data satisfying

$$
N\left[\frac{\mu T^{2}}{2}+\frac{\left(1-p^{2}\right)^{2}}{4}, 1\right]\left(0^{+}\right)-\frac{\lambda^{2}}{2 N}\left[p, \Delta_{N} p\right]\left(0^{+}\right)-\frac{\alpha a^{2}}{2}\left[T, \Delta_{N} T\right]\left(0^{+}\right)=O(1) .
$$

Interesting data satisfying (3.16) may be achieved in a variety of ways. Below we outline one such construction. We let $\Gamma$ be a simple closed curve of finite arclength $L(\Gamma)$ which is wholly contained in $\Omega, \mathcal{S}_{c}$ be all cells $\Omega_{i, j}$ which are cut by $\Gamma, \mathcal{S}_{I}$ be all cells $\Omega_{i, j}$ which are interior to the region surrounded by $\Gamma$, and $\mathcal{S}_{E}$ be those cells $\Omega_{i, j}$ which are in $\Omega$ and exterior to $\Gamma$. For definiteness we choose

$$
p_{i, j}\left(0^{+}\right)= \begin{cases}1, & \text { if } \Omega_{i, j} \in \mathcal{S}_{E} \\ p_{i, j}^{*} \in[-1,1], & \text { if } \Omega_{i, j} \in \mathcal{S}_{c} \\ -1, & \text { if } \Omega_{i, j} \in \mathcal{S}_{I}\end{cases}
$$

and

$$
T_{i, j}\left(0^{+}\right)=h_{0}-\left[\left(p-p^{3} / 3\right)\left(0^{+}\right), 1\right]=O\left(1 / N^{1 / 2}\right) .
$$

Such data models the situation where initially water surrounds ice. With such data it is easily checked that $N\left[T^{2}\left(0^{+}\right), 1\right]=O(1)$, while $N\left[\left(1-p^{2}\right)^{2}, 1\right]$ and $-\frac{1}{N}\left[p, \Delta_{N} p\right]$ are $O(L(\Gamma))$ and thus the functional defined in (3.16) is $O(1)$ independently of $N$. For such data we note that $\delta^{3}\left[\Delta_{N} p, \Delta_{N} p\right]=\frac{1}{N^{3}}\left[\Delta_{N} p, \Delta_{N} p\right]$ is also $O(L(\Gamma))$ independently of $N$. The last functional enters in the discrete analogue of (2.68).

The simulations we present were all run with the following parameters:

$$
(\alpha, \lambda, a, \mu)=(1,1,1,1)
$$

We chose $N=200$

$$
\mathrm{d} x=\mathrm{d} y=\delta=1 / 200
$$


and

$$
\mathrm{d} t=\mathrm{d} x / 10 .
$$

With these parameters

$$
m 1 \stackrel{\text { def }}{=} \mathrm{d} t /(\mathrm{d} x)^{2}=10
$$

and

$$
m 2 \stackrel{\text { def }}{=} \frac{\delta \mathrm{d} t}{(\mathrm{~d} x)^{2}}=\frac{1}{10}
$$

and thus we were compelled to use an implicit integration scheme for (3.10). Our choice was ADI working with the fixed tri-diagonal matrix whose non-zero entries are

$$
A(i, i)= \begin{cases}1+m 1 & \text { if } i=1 \text { and } i=200 \\ 1+2 m 1 & \text { if } 2 \leq i \leq 199\end{cases}
$$

and

$$
A(i, i+1)=A(i+1, i)=-m 1, \quad 1 \leq i \leq 199
$$

We used a first order explicit Euler scheme on (3.11).

Our first simulation was run with the following choice of initial data:

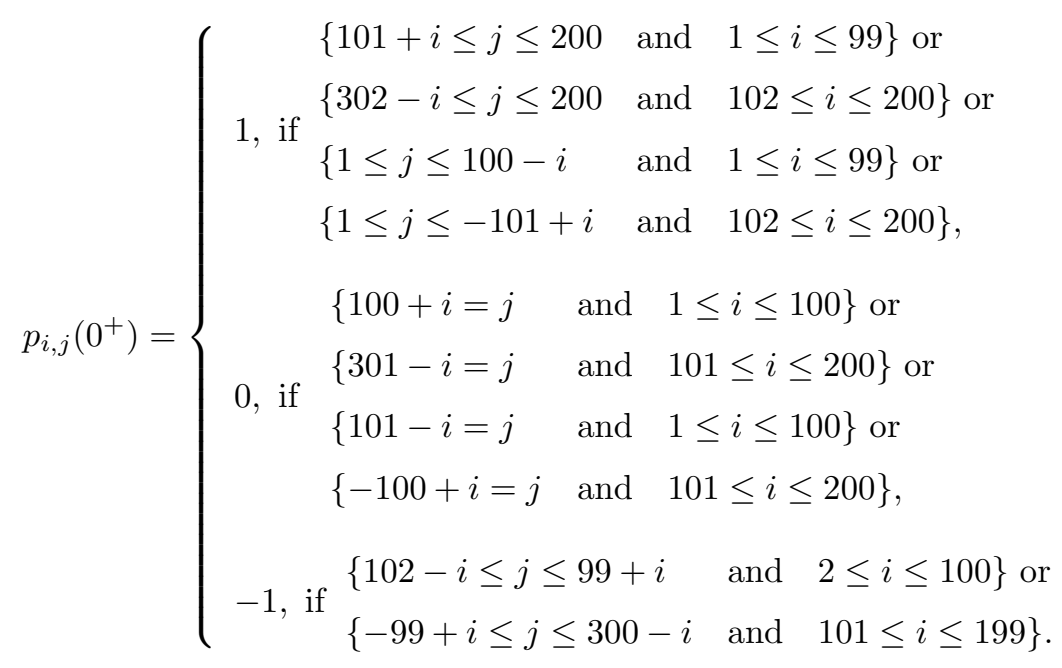

This data satisfies $[p, 1]=\left[p^{3}, 1\right]=0$. We chose $h_{0}=0.05$ and $T_{i, j}\left(0^{+}\right) \equiv 0.05,1 \leq i, j \leq 200$.

The initially superheated square block of ice converged to a circular block centered at $(0,0)$ with a radius consistent with the $\delta=0^{+}$asymptotics. Specifically, if we grant that the "equilibrium" curve separating the ice from the water is a circle of radius $R$, then the "Gibbs-Thomson" relation (1.6), with our choice of parameters, implies that

$$
\frac{0.005}{2^{1 / 2} R}=-T_{M}
$$


while (3.12) implies that

$$
T_{M}=0.05-\left[p-p^{3} / 3,1\right]
$$

Noting that $p_{i, j} \simeq-1$ inside of the circle and $p_{i, j} \simeq+1$ outside yields

$$
\left[p-p^{3} / 3,1\right]=\frac{2}{3}\left(1-2 \pi R^{2}\right)
$$

and (3.27)-(3.29) imply that $R$ satisfies

$$
\frac{4 \pi}{3} R^{2}+\frac{0.005}{2^{1 / 2} R}=\frac{2}{3}-0.05
$$

(3.30) has one positive solution close to zero and a second larger solution $R=0.3808$ which is the approximate equilibrium radius we obtain in our simulation. This radius, when substituted into the Gibbs-Thomson relation (3.27) yields a melt temperature consistent with what we observe. The results of this simulation are shown in Figure 1.

Our final simulation starts with 16 regularly spaced circular blocks of ice surrounded by water. Specifically

$$
p_{i, j}\left(0^{+}\right)= \begin{cases}-1, \text { if } & \sqrt{(i-40 m)^{2}+(j-40 n)^{2}} \leq 15 \\ & \text { and } 1 \leq m, n \leq 4 \\ 1, & \text { otherwise }\end{cases}
$$

For this data

$$
\left[\left(p-p^{3} / 3\right)\left(0^{+}\right), 1\right]=0.2885 .
$$

We again chose $h_{0}$ to be 0.05 and at $t=0$ took the uniform temperature field

$$
T_{i, j}\left(0^{+}\right)=h_{0}-\left[\left(p-p^{3} / 3\right)\left(0^{+}\right), 1\right]=-0.2385 .
$$

The solutions using this initial data converged to the same equilibrium as before but the approach was far more interesting. The solutions at times $t=0.05,0.17,0.24,0.4,0.6,0.7,2,3.3$, and 12.5 are shown in Figures 2-10.

We also ran both sets of data with the "intermediate" model and obtained virtually identical results.

\section{Explanation of Graphics}

In Figures 1-10 the upper left frame is a contour plot of $p$ and $p$ is 1 in a neighborhood of the boundaries $x= \pm 1 / 2$ and $y= \pm 1 / 2$ and the upper right hand frame is a contour plot of $T$. In Figure 1 the lower left plot shows $p, T$ and $p-p^{3} / 3+T$ along the lines $x=0, y=0$, and the $45^{\circ}$ diagonal through the origin. These plots overlay one another because of the circular symmetry of the final state. In Figure 1 the bottom right plot again displays the same information. In Figures $2-10$ the bottom left plot shows $p, T$, and $p-p^{3} / 3+T$ along the $45^{\circ}$ diagonal through the origin and the bottom right plot shows the same quantities on the line $x=0$. The reader interested in seeing a movie of the second simulation should consult reference 3 on the author's web site: http://www.math.cmu.edu/math/people/greenberg.html. 

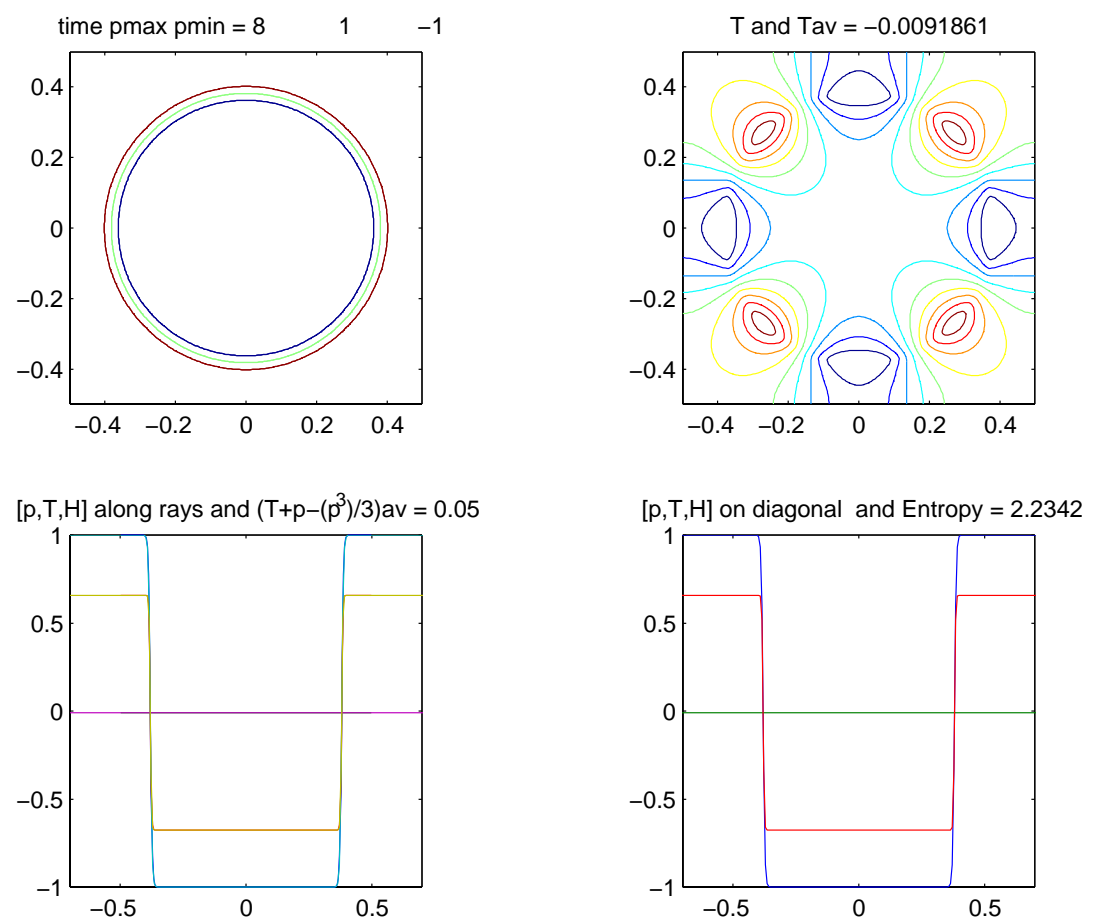

Figure 1.
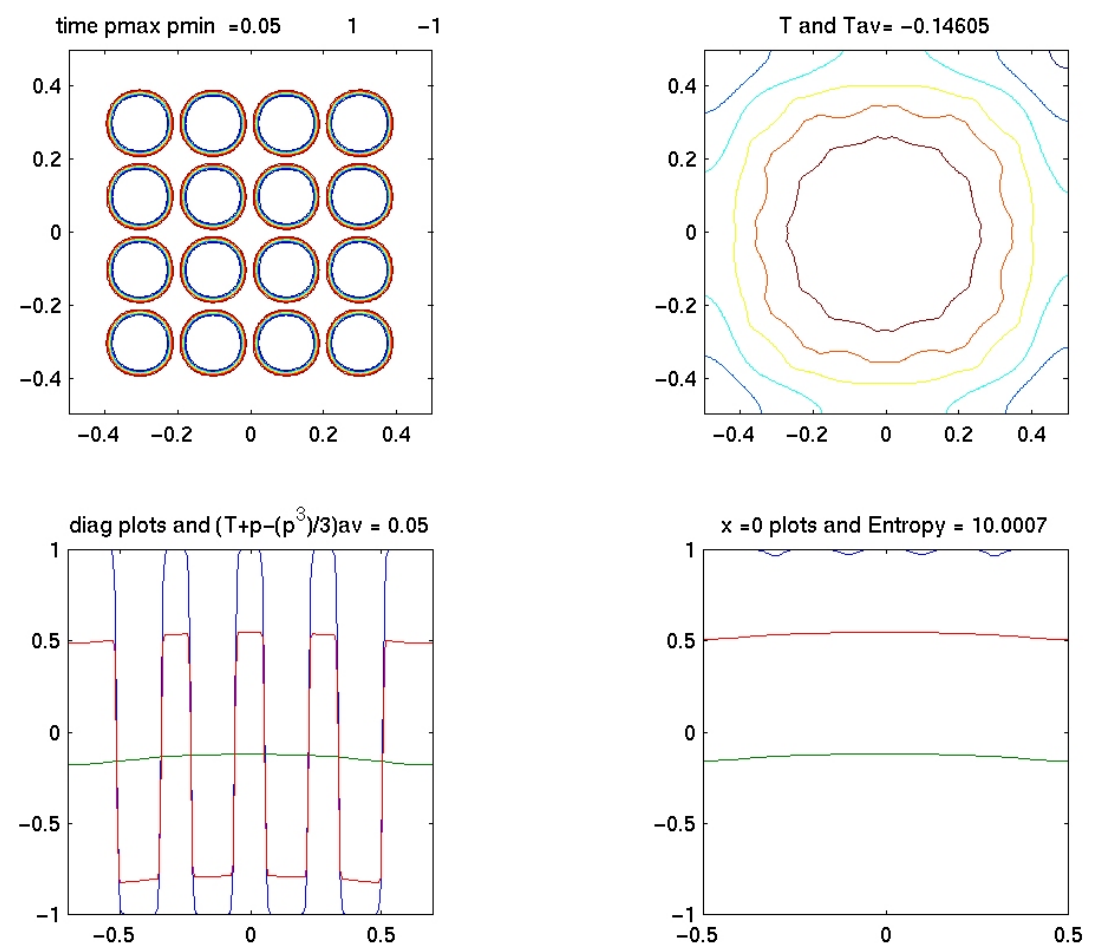

FIGURE 2. 

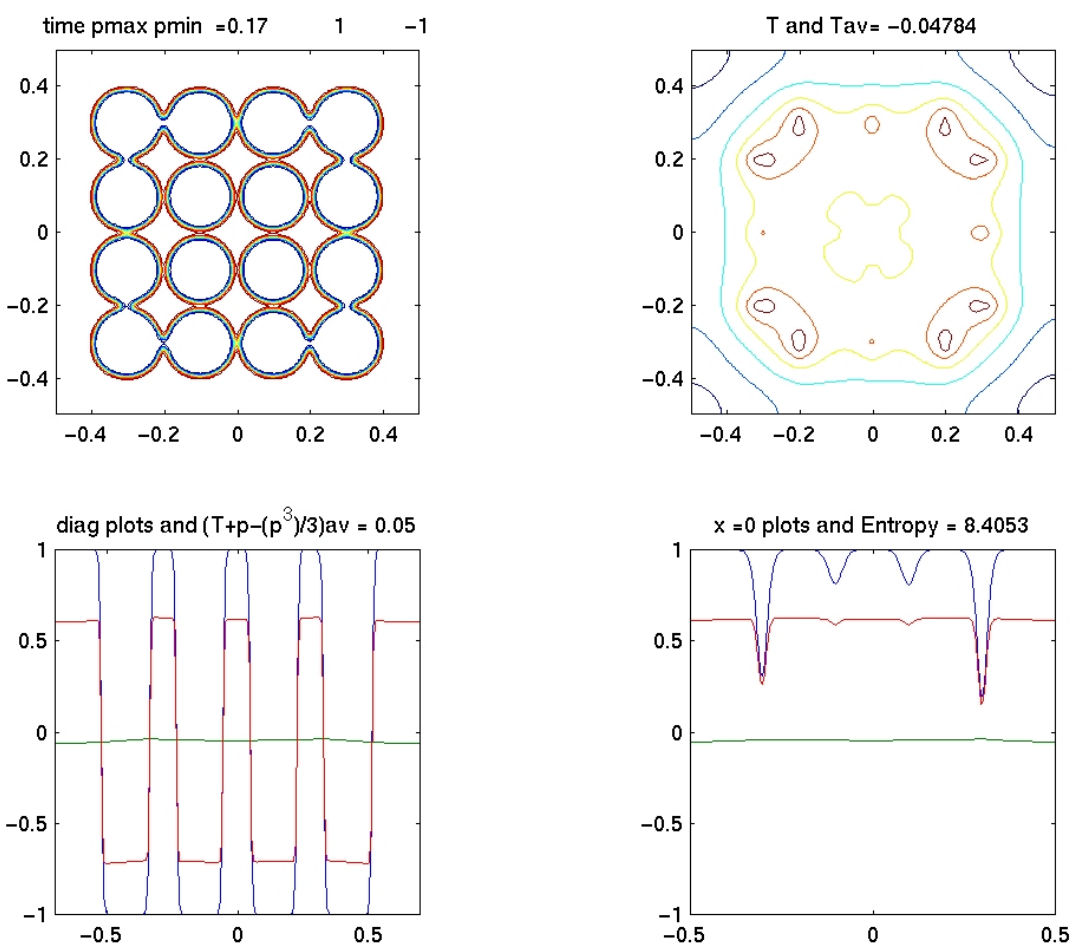

Figure 3.
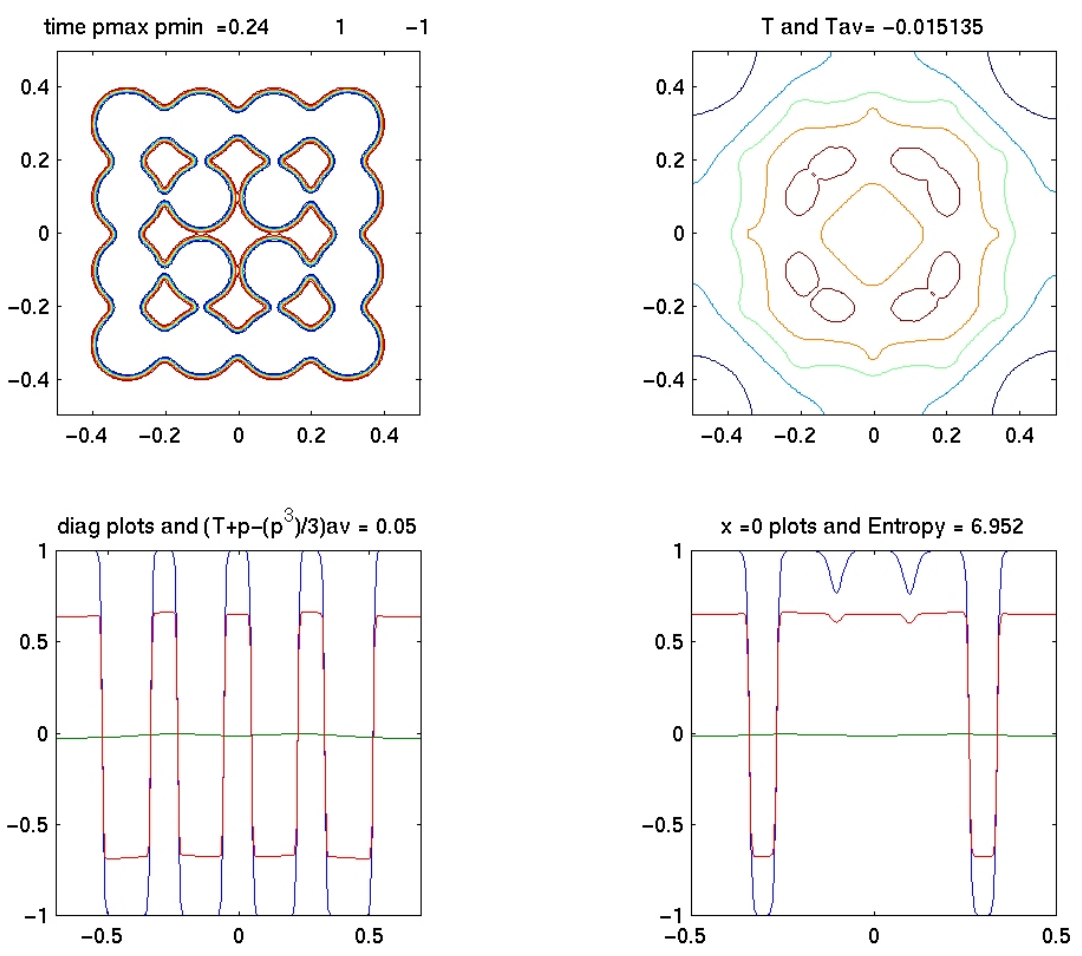

Figure 4. 

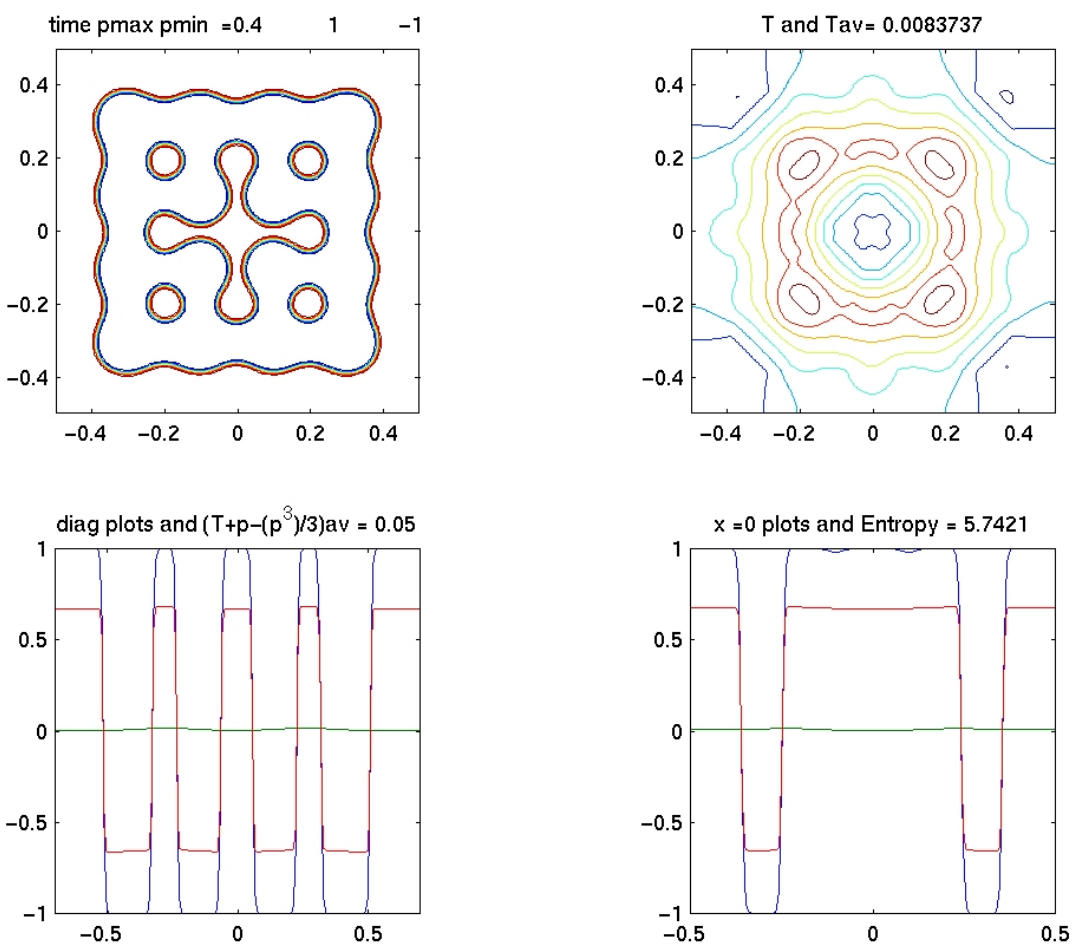

FIGURE 5.
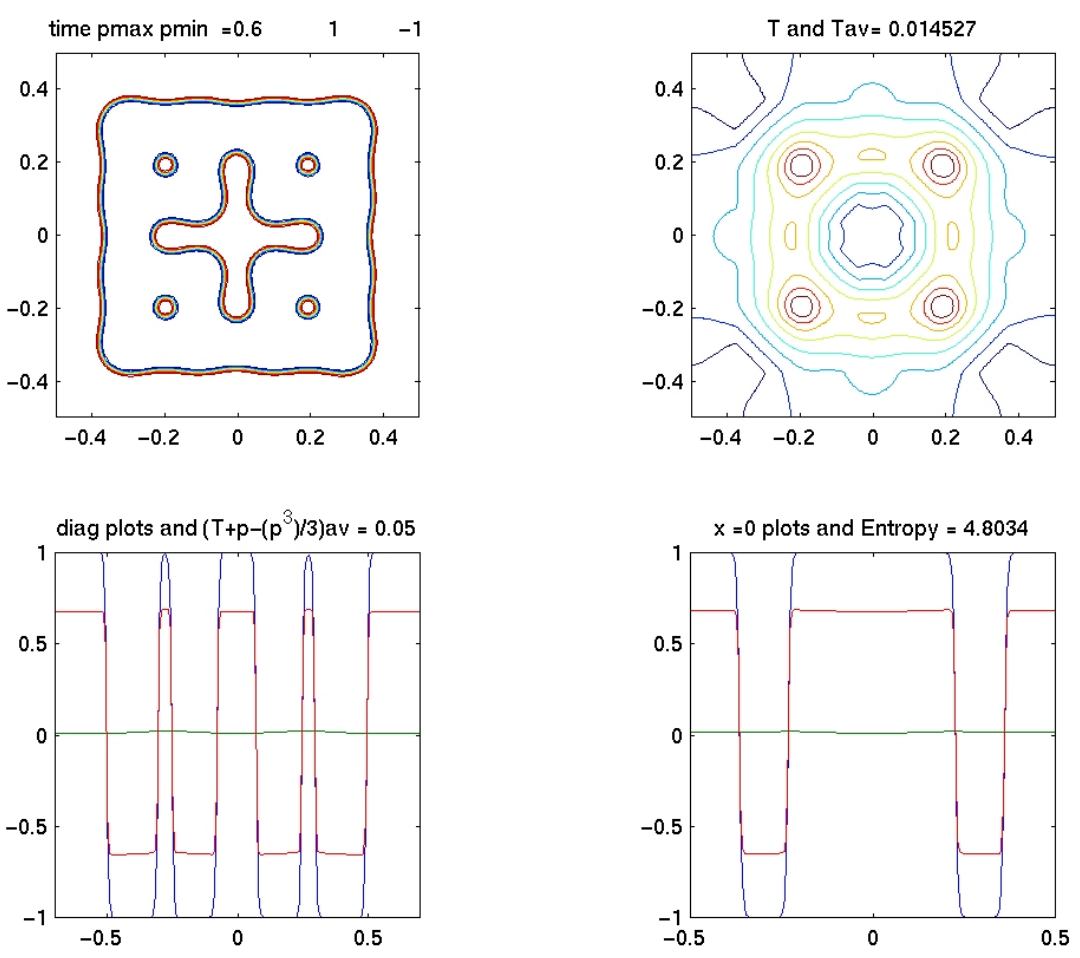

FiguRE 6 . 

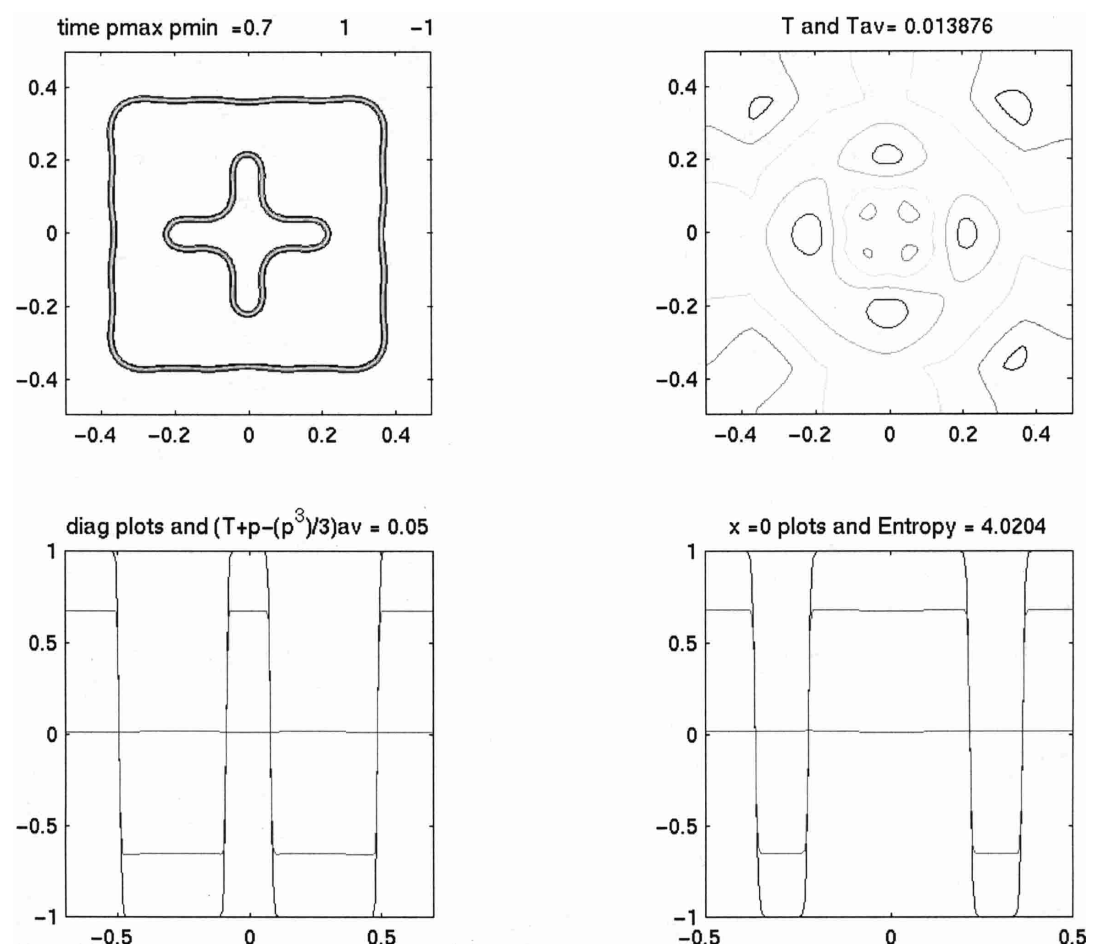

Figure 7.
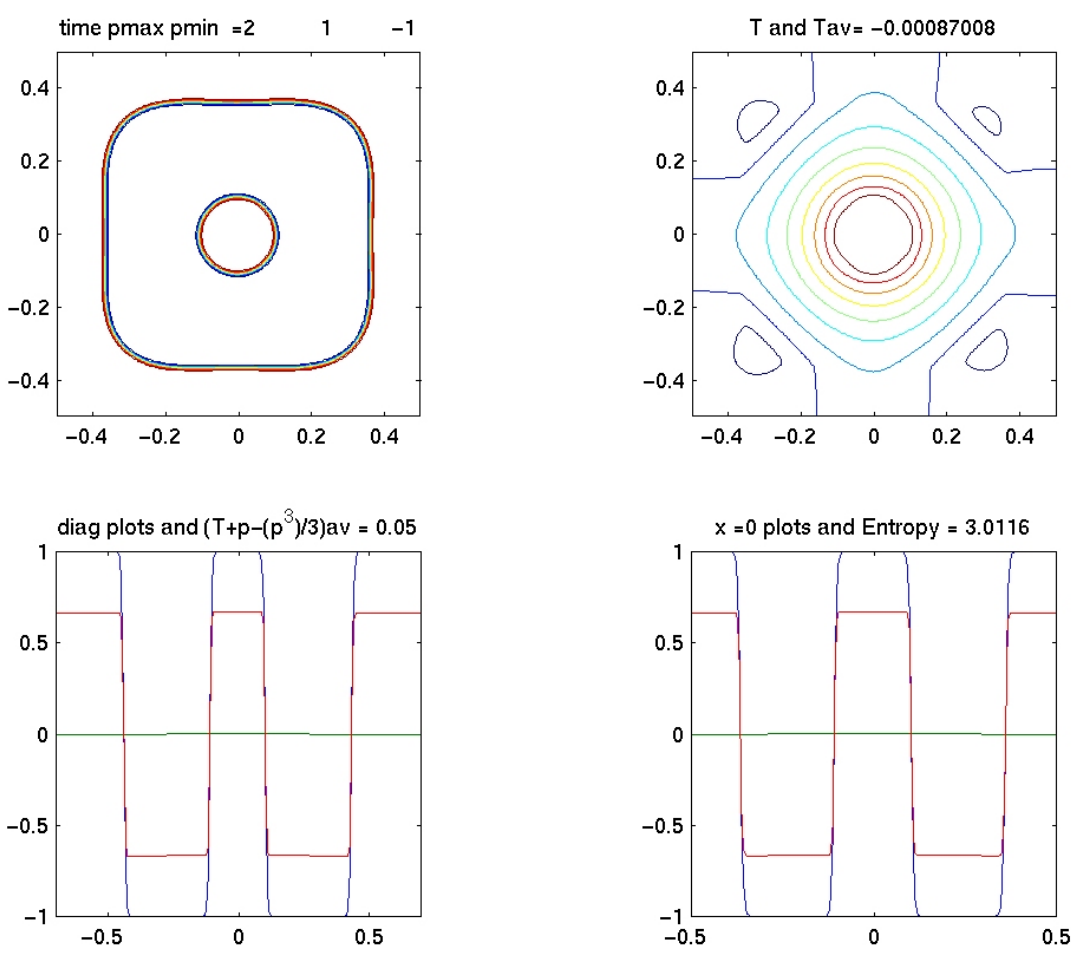

Figure 8. 

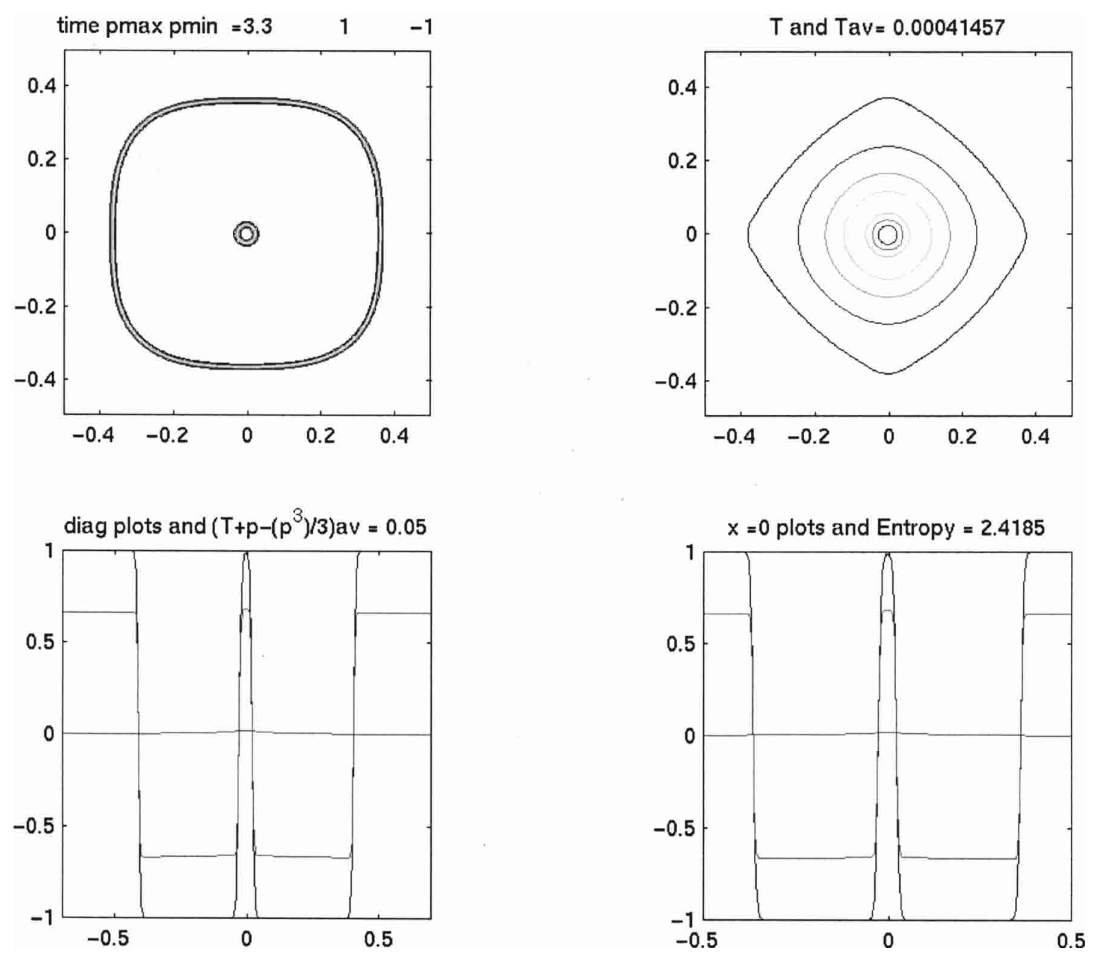

Figure 9
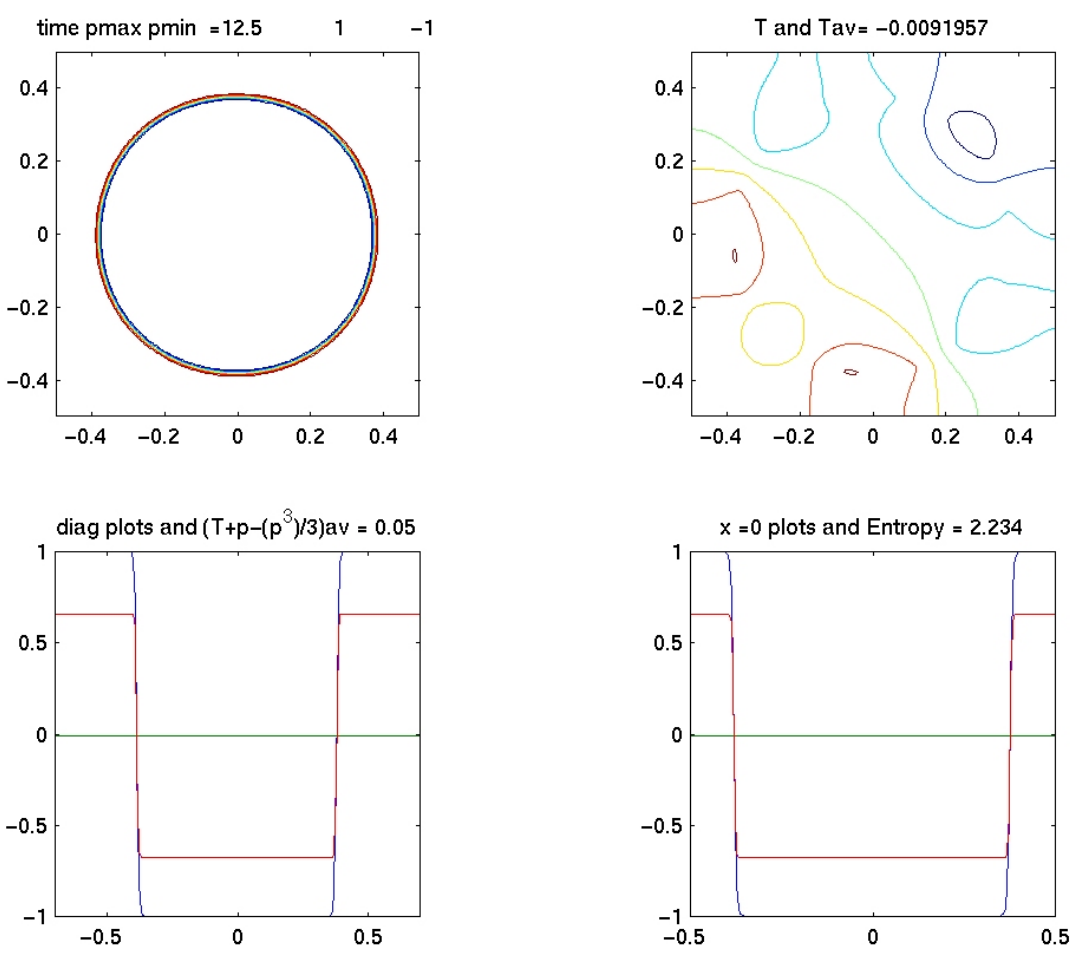

Figure 10. 


\section{Concluding Remarks}

We have established the existence of properly scaled entropies of successively higher order in the space and time derivatives of $T$ and $p$ which are bounded on solutions of (1.1) and (1.2). These entropies yield information (i) about the sharp-interface, $\delta=0^{+}$limits of solutions of (1.1) and (1.2) and (ii) large $t$ information about the solutions of (1.1) and (1.2) at fixed $\delta$. More importantly we have shown correspondingly spatially discrete entropies for the spatially discretized version of this system which are faithful to the entropies of the continuous system.

We have carried out the calculations to verify that the system (1.4) and (1.5) with $m=0$, which has been studied extensively by Caginalp [1-3], is endowed with similar entropies. As noted earlier, this system is not endowed with the pointwise estimates $-1 \leq p(x, y, t) \leq 1$, but the fact that the temperature difference, $T$, appears linearly in (1.5) saves the day. We have not succeeded in carrying out our program of rigorous estimates for (1.4) and (1.5) when $n=m \geq 2$.

Perhaps the most important /interesting conclusion of this study is that under mild and natural conditions on the initial data the solutions of (1.1) and (1.2) satisfying $\frac{\partial T}{\partial n}=\frac{\partial p}{\partial n}=0$ on $\partial \Omega$ asymptotically evolve as solutions of the reduced system (1.10), (1.11), and (1.13).

\section{REFERENCES}

[1] G. Caginalp, An analysis of a phase-field model of a free boundary. Arch. Rat. Mech. Anal. 92 (1986) $205-245$.

[2] G. Caginalp, Stefan and Hele-Shaw type models as asymptotic limits of the phase field equation. Phys. Rev. A 39 (1989) 5887-5896.

[3] G. Caginalp, Phase field models and sharp interface limits: some differences in subtle situations. Rocky Mountain J. Math. 21 (1996) 603-616.

[4] G. Caginalp and X. Chen, Phase field equations in the singular limit of sharp interface problems, in On the evolution of phase boundaries, IMA 43 (1990-1991) 1-28.

[5] G. Caginalp and E. Sokolovsky, Phase field computations of single-needle crystals, crystal growth, and motion by mean curvature. SIAM J. Sci. Comput. 15 (1994) 106-126.

[6] M. Fabbri and V.R. Vollmer, The phase-field method in the sharp-interface limit: A comparison between model potentials. J. Comp. Phys. 130 (1997) 256-265.

[7] G.B. McFadden, A.A. Wheeler, R.J. Brown, S.R. Coriell and R.F. Sekerka, Phase-field models for anisotropic interfaces. Phys. Rev. E 48 (1993) 2016-2024.

[8] O. Penrose and P. Fife, Thermodynamically consistent models of phase-field type for the kinetics of phase transitions. Physica D 43 (1990) 44-62.

[9] O. Penrose and P. Fife, On the relation between the standard phase-field model and a "thermodynamically consistent" phasefield model. Physica D 69 (1993) 107-113.

[10] S.L. Wang, R.F. Sekerka, A.A. Wheeler, B.T. Murray, S.R. Coriell, R.J. Braun and G.B. McFadden, Thermodynamicallyconsistent phase-field models. Physica D 69 (1993) 189-200.

[11] S.L. Wang and R.F. Sekerka, Algorithms for phase field computations of the dendritic operating state at large supercoolings. J. Comp. Phys. 127 (1996) 110-117.

To access this journal online:

www.edpsciences.org 